\title{
Linx
}

Revue des linguistes de l'université Paris X Nanterre

$50 \mid 2004$

Variation sémantique et syntaxique des unités

lexicales : étude de six verbes français

\section{Syntaxe, référence et identité du verbe filer}

\section{Sarah de Vogüé}

\section{(2) OpenEdition}

Journals

Édition électronique

URL : http://journals.openedition.org/linx/142

DOI : 10.4000/linx.142

ISSN : 2118-9692

Éditeur

Presses universitaires de Paris Nanterre

\section{Édition imprimée}

Date de publication : 1 juin 2004

Pagination : 135-167

ISSN : 0246-8743

\section{Référence électronique}

Sarah de Vogüé, «Syntaxe, référence et identité du verbe filer », Linx [En ligne], 50 | 2004, mis en ligne le 27 janvier 2011, consulté le 21 avril 2019. URL : http://journals.openedition.org/linx/142 ; DOI : 10.4000/linx.142 


\title{
Syntaxe, référence et identité du verbe filer
}

\author{
Sarab de Vogüé \\ Université Paris $X$ - Nanterre \\ UMR 7110 (Université Paris 7)
}

Le verbe filer fait l'objet d'une variation relativement importante, tant sur le plan des conditions d'emploi qui sont les siennes, que sur celui de ses valeurs.

Quatre grandes catégories de constructions syntaxiques peuvent être répertoriées : des constructions intransitives (Le temps a filè), des constructions transitives (J'ai filé mon bas), une construction avec structure prépositionnelle filer GP + ((pour) Infinitif) où $\mathrm{P}$ est une préposition de localisation (Il a filé chez elle pour lui parler), une construction avec structure attributive de type filer $G N$ à $G N^{1}$ (Ils ne veulent pas me filer ma moto).

En termes d'environnement lexical, on trouve des co-textes non quelconques mais nettement diversifiés, manifestant des relations de sélection argumentale variées : on va de l'araignée au fugitif en position sujet, en passant par le caramel, le navire, le chemin, le bas ou la maille, la lampe, le pouls, l'argent et la vie ; en position objet, on trouve des matières textiles, des humains, à nouveau le bas ou la maille, des pièces de théâtre, des sons, des destins, mais aussi, dans la construction attributive, des objets, prêtés ou donnés, des sentiments, des coups, des maladies.

En termes de valeurs, il est question de production de fils, filage ou filature, mais aussi de filatures dans l'autre sens du mot (filer Mesrine); il est question de déroulements (filer un câble, une maille, un bas) où quelque chose se dévide ou se défait ; il est question de disparitions (il a filê), ou seulement d'un déplacement dans l'espace (filer à l'école), éventuellement de sa rapidité, quand il ne s'agit pas d'une simple façon d'occuper l'espace (le chemin qui file à droite), ou de se dérouler dans le temps (voir la continuité que dit le filage de la pièce, la tenue du son qu'on file, la faiblesse du pouls

\footnotetext{
${ }^{1}$ Evelyne Saunier (communications au séminaire «Invariants langagiers » mentionné note 6) signale aussi quelques emplois où le verbe est dans une construction «mixte», à la fois attributive et locative filer $G N+G P$, qu'elle a recensés en nombre non négligeable dans un corpus de romans policiers.
} 
qui file) ; il est question aussi, dans les constructions attributives, de différentes formes de transmission.

Une telle diversité n'a en soi rien d'exceptionnel. Elle est cependant suffisamment développée pour constituer un bon terrain empirique pour une mise à l'épreuve des différents principes de variation dont fait l'hypothèse le programme de recherche sur l'identité lexicale issu de la Théorie des Opérations Prédicatives et Enonciatives (TOPE) qui s'est élaborée autour des travaux d'Antoine Culioli. On se propose ainsi de montrer le rôle décisif qui peut être joué par des variations relatives au caractère plus ou moins qualitatif et plus ou moins quantitatif des référents décrits. Ce sera l'occasion de redéployer le principe de ces variations, où s'opposent ce que l'on a l'habitude d'appeler dans la TOPE le « discret», le « dense » et le « compact». On se propose par ailleurs de donner des éléments de réflexion sur les différentes formes que peuvent prendre la variation selon qu'elle relève de principes généraux, ou d'une simple adaptation au contexte. On se propose enfin de montrer comment la syntaxe intervient dans la diversification des emplois et des valeurs, en reprenant deux outils élaborés indépendamment dans le cadre de la TOPE : d'une part des hypothèses sur différentes formes de relations syntaxiques possibles entre unités, venant complexifier une perspective strictement compositionnelle sur la combinatoire des unités (combinaison par ajout, par greffe ou par nouage); d'autre part des hypothèses sur l'articulation de plusieurs niveaux dissociés de structuration de l'identité lexicale (forme schématique, schéma grammatical, schémas de complémentation), venant compliquer une approche strictement distributionnelle. Cela nous conduira à revenir sur des notions syntaxiques centrales: celle de transitivité en particulier, dont on montrera comment elle peut être construite à partir de structures intransitives; celle de catégorie grammaticale, quand il s'avérera que fil et filer sont fondamentalement identiques dans leur fonctionnement sémantique, ne différant pour l'essentiel que par leur statut dans l'économie générale de l'énoncé.

Étant donnée la dimension de l'article, étant donné aussi le nombre relativement important de questions générales que cette analyse doit traverser, on ne pourra pas mesurer dans le détail la façon dont ces différents facteurs de diversification génèrent la variation observée. On se contentera donc d'indiquer la forme que prend cette diversification, en laissant le soin à une étude plus longue et plus développée de tester plus précisément les effets de chacun des principes de variation mentionnés, et de modifier ce faisant le détail ou le contenu même des analyses proposées.

Le point de départ de l'analyse sera en revanche beaucoup plus développé. Il consiste à déterminer les différents ingrédients que peut convoquer le mot, et à montrer comment il les configure : on restitue ce faisant ce que l'on décrit comme étant la «forme schématique» de filer, qui est censée figurer ce qui fait l'identité singulière de ce mot. Il s'avérera que cette forme schématique est globalement indifférente à la syntaxe du mot. Reste qu'elle est foncièrement relationnelle, se définissant comme une forme de schématisation de ce qui peut constituer le contexte de filer. On l'élabore en formalisant pour chaque type d'emploi ce que Benveniste a décrit comme étant la «fonction intégrative» du mot, c'est-à-dire la façon dont il détermine un co-texte et l'intègre dans un schéma de signification, pour « effectuer la signification » du tout dont il est le noyau. Cela suppose un parcours systématique de 
tous ces emplois dans chacune des facettes de leurs valeurs. Un tel parcours est nécessairement un peu long.

L'investigation à laquelle donne lieu cette recherche de l'identité du mot est d'ordre fondamentalement linguistique : on s'intéresse aux valeurs du mot dans les énoncés, à ses contextes d'emploi, à la façon dont ces contextes sont configurés. Il demeure qu'elle touche, ne serait-ce qu'à travers les ingrédients qui se trouveront ce faisant mis en évidence, à ce qui pourrait constituer le référent du mot, qui lui est d'ordre extra-linguistique. On se voit donc nécessairement confrontés à la difficile question des relations entre sens et référence.

De fait, avec un mot comme filer, la séparation entre sens et référence est particulièrement délicate. D'abord parce que ce mot a toutes les caractéristiques des mots que l'on décrit traditionnellement comme concrets, dont la valeur s'entend comme devant être un type de référent concret. Ensuite, parce que l'ensemble de ses valeurs parait s'organiser autour d'une valeur première, qui pourrait tenir lieu de sens propre, dans laquelle le mot fait figure de terme technique pour désigner une activité bien identifiée sur le plan cognitif: l'activité consistant à transformer en fil quelque matière textile.

Les théories du sens qui organisent les valeurs des mots autour d'un sens propre sont des plus répandues et des plus anciennes. Ce sont souvent les mêmes théories qui militent pour que le sens soit traité comme une simple rampe d'accès à un contenu référentiel externe à la langue. Il est fréquent en outre qu'on associe les deux démarches, en choisissant pour sens propre les emplois où le référent est le plus concret possible. Ce type d'approche, contre laquelle Benveniste a ferraillé longuement en son temps ${ }^{2}$, a retrouvé une forme de modernité au travers des positions de tenants importants du cognitivisme. Que ce soit pour un cognitiviste contemporain comme Lakoff ou pour les étymologistes les plus conservateurs hérités du XIX ${ }^{e}$ siècle, le sens concret vient d'abord. Quant à la thèse selon laquelle on pourrait rendre compte du sens sinon à l'aide du référent, du moins à partir des propriétés cognitives de ce référent, elle est au fondement même de la démarche cognitive en sémantique.

Ce ne sont pas les positions qui seront défendues ici et qui sont défendues de manière générale dans les analyses lexicales qui ont pu être menées au sein du programme de recherche sur les formes schématiques. On s'opposera à la solution «propre» qui place telle valeur concrète de «filage» au centre des emplois de filer: cette valeur correspond en l'occurrence à un emploi transitif du verbe, avec d'un côté un fileur, de l'autre une matière filée, et on soutiendra que les emplois transitifs loin d'être premiers sont construits. On s'opposera aussi aux solutions «cognitives » qui tenteraient de rendre compte des valeurs et du fonctionnement de filer en se fondant sur les propriétés cognitives du référent, c'est-à-dire sur la façon dont ce référent est conceptuellement construit. Non pas que ces propriétés soient inintéressantes, non pas même que la langue n'en soit pas informée, non pas qu'elle ne s'attache pas au

\footnotetext{
${ }^{2}$ Voir en particulier Benveniste (1954), dans lequel Benveniste s'oppose au privilège que d'aucuns voudraient accorder aux valeurs concrètes. On y trouve des rappels à l'ordre importants à adresser encore à la communauté des sémanticiens, pour ne pas oublier «combien est importante la distinction nécessaire entre la signification et la désignation » (p. 301).
} 
moins partiellement à restituer ces propriétés ${ }^{3}$. Mais on soutiendra, au nom de l'arbitraire des langues, que celles-ci ne sauraient se réduire à être l'expression de constructions cognitives élaborées indépendamment qui en donneraient la raison ${ }^{4}$. Les langues élaborent leurs propres conceptualisations du monde, en relation sans doute avec les conceptualisations qui s'élaborent par ailleurs, à travers la perception, à travers la culture, à travers les pratiques, mais sans être vouées à simplement les refléter. En nommant filer la pratique du filage, la langue dit sans doute cette pratique, mais elle la dit à sa guise, à travers le tissu de relations qu'instaure le système dans lequel le mot filer est pris ${ }^{5}$.

Reste que si l'on a choisi de s'intéresser ici à un mot comme filer, c'est bien à cause de la façon dont avec un tel mot le référent parait s'inviter dans la langue ${ }^{6}$. Il

3 On n'adhère donc pas ici à une version stricte du constructivisme que Cadiot et Lebas (2003) prêtent aux culioliens : les valeurs référentielles que la langue construit ne sont pas opaques par rapport à un référent extralinguistique autonome; elles sont des reconstructions de ce référent. On s'intéresse donc aux lits (ceux des rivières, celui du fascisme, ceux où l'on dort) et aux fils et à la grandeur quand on doit décrire la valeur du mot lit, du mot filer et du mot grand.

${ }^{4}$ On s'oppose donc ici aux positions de Cadiot et Lebas (2003), positions d'ailleurs partagées en général par les tenants de la sémantique cognitive. S'il y a sans doute « constitution extrinsèque » des objets, cette constitution extrinsèque ne saurait fournir la clef des mots, que ceux-ci n'auraient alors plus qu’à nommer et qui rendrait compte de leur richesse sémantique.

Ce qui est contesté ici est l'idée de «l'extraordinaire transposabilité de tout ce qui peut faire sens » que viendraient prouver de manière abondante les «travaux de la psychologie». Qu'il y ait des phénomènes de cohérence plus ou moins locale entre les systèmes de sens est certain, ce qui permet de rabouter un sens à l'autre, mais on ne voit pas pourquoi le fil de la brodeuse devrait être une transposition du fil de la couturière, que le mot fil lui-même ne ferait que transposer. On s'oppose donc à cette psychologie-là, comme à l'idée d'un sens « nécessairement transversal » qui serait le sens que les mots n'auraient alors plus qu'à dire avec leurs moyens propres de mots. Il n'y a pas nécessairement concordance en particulier parce que la réalité du fil, et de manière plus générale la réalité des référents, reste de toutes façons extérieure à toutes ces conceptualisations qui tentent de la cerner mais auxquelles toujours elle résiste. De fait, au modèle gestaltiste des correspondances on oppose le modèle lacanien du nœud borroméen : les conceptualisations (ce que Lacan désigne par la lettre I pour imaginaire), les inscriptions symboliques dans le tissu d'une langue (ce qui correspond à ce que Lacan désigne par la lettre $\mathrm{S}$ pour symbolique), et le réel (la lettre $\mathrm{R}$ ), sont simplement des cercles en intersection, qui se tiennent mutuellement (plus précisément le nœud fait que chacun tient ensemble les deux autres, et que si l'un est rompu les deux autres se séparent), mais qui restent autonomes.

5 Pour illustrer cela, on peut prendre un autre exemple : dans de Vogüé et Franckel (2002), nous soutenons que grand met toujours en jeu une frontière (pour dire alors qu'il y a de l'espace avant d'atteindre cette frontière), cette frontière pouvant par ailleurs prendre différentes statures, parfois bord, parfois norme. Or, si l'on considère tous les objets grands du monde, il n'y aurait aucun sens à trouver dans tous la frontière en question: car c'est le mot qui pose l'objet par rapport à une frontière, et il n'est pas nécessaire que l'objet présente en lui-même une telle frontière.

${ }^{6}$ C'est à ce titre que ce verbe a été choisi il y a quelques années comme objet d'étude commun pour le séminaire du groupe «Invariants langagiers » du Laboratoire de Linguistique Formelle. L'analyse qui suit s'appuie en particulier sur les discussions, les recherches et les propositions qui ont été émises dans ce cadre. Elle a de ce fait bénéficié du travail de Rémi Camus, Antoine Culioli, JeanJacques Franckel, Denis Paillard, Bernard Victorri, Evelyne Saunier, même si ceux-ci ne sont pas 
s'agit de comprendre comment la langue, en disant le référent, le reconstruit. Il s'agit de prendre la mesure de cette forme de savoir sur le monde que la langue se trouve ainsi déployer. On voit qu'il s'agit encore de cognition, mais d'une cognition inscrite dans la langue: le témoignage que la langue nous donne ${ }^{7}$ de ce que peut être une filature, de ce que peut être un fil, de ce que ce peut être pour du caramel ou pour Mesrine que de filer.

\section{La syntaxe de filer: le répertoire de ses structures de complémentation}

On considère d'abord le plan des constructions syntaxiques. On a vu qu'elles étaient variées. Une telle diversité n'est pas spécifique à filer: contrairement à ce qu'on a pu penser au moment où émergea l'idée d'identifier une unité par son type de distribution, il est fréquent qu'une même unité entre dans plusieurs distributions syntaxiques. Sauf à pouvoir réduire ces distributions les unes aux autres, une approche distributionnaliste stricto sensu devrait en conclure à l'existence d'homonymes. Or, dans le cas qui nous occupe, il est quelques emplois qui sont de faux intransitifs et s'analysent comme des transitifs absolus (par exemple quand Paul filait signifie que Paul filait tel ou tel matériau textile); il est quelques cas aussi où la construction prépositionnelle peut être restituée derrière une structure sans GP apparent (quand Pierre a filé sa bagnole se trouve rapporté à une construction attributive par exemple). Mais on ne trouve pas de dérivation simple de ce type dans la plupart des cas. Pourtant la solution de l'homonymie est inacceptable : entre les diverses valeurs obtenues, on observe une forme de proximité sémantique qui conduit à admettre qu'il doit y avoir dans ce que dit filer quelque chose qui reste indépendant des constructions particulières dans lesquelles il s'inscrit.

On l'admettra d'autant mieux que les constructions en question, très générales, ne sauraient être tenues en elles-mêmes pour spécifiques de filer: on peut concevoir que le co-texte lexical soit dans sa configuration même propre à filer, en revanche des constructions syntaxiques comme la construction transitive ou la construction intransitive, ou même tel ou tel sous-type de construction intransitive, recouvrent par définition des classes entières de lexèmes (les «sous-catégories» de la théorie de la sous-catégorisation) et doivent par conséquent s'entendre comme étant fondamentalement extérieures à ce qui fait l'identité du mot. C'est dire que ce qui fonde l'identité singulière du mot et ce qui se donne comme le répertoire de ses constructions syntaxiques, doivent être séparés.

En même temps, le répertoire en question participe à la caractérisation du mot. On peut même supposer que chaque lexème impose des contraintes sur les types de constructions qu'il admet, ces contraintes étant alors partie prenante de ce qui fait son

pour autant responsables de ce qu'a pu devenir leur travail une fois intégré dans le cadre qui était le mien.

${ }^{7}$ Un savoir comme un autre, important, qui peut intéresser les cognitivistes, qui intéresse parfois les philosophes, quand pour comprendre un concept, ou une donnée du monde, ils cherchent à comprendre ce que la langue en dit, c'est-à-dire ce que disent en particulier les mots qui nomment ce concept ou cette donnée. 
identité. Il faut dès lors admettre que ces constructions sont à la fois internes et externes au lexème : elles sont ce qui dans la caractérisation de ce lexème l'apparente à d'autres lexèmes; elles sont ce par quoi le lexème se trouve inscrit dans diverses classes de lexèmes, par delà ce qui fait sa singularité.

On doit par conséquent donner une représentation du lexème dans laquelle elles interviennent, non pas comme définitoires de l'identité du lexème, mais comme participant à sa caractérisation. On doit alors distinguer au moins deux niveaux dans l'identification de ce lexème, un niveau l'identifiant dans sa singularité, et un niveau l'incluant dans différentes sous-catégories, et donnant donc la liste des différentes structures de complémentation qu'il admet.

Si ces deux niveaux sont distincts, cela signifie que l'une des tâches de l'analyse sera de rendre compte des structures de complémentation en question : il s'avère que caractériser le lexème dans sa singularité ne suffit pas pour ce faire.

Mais cela signifie aussi que l'on peut dans un premier temps s'attacher à reconstituer cette singularité en faisant abstraction des structures syntaxiques impliquées. Il faut alors que l'on ait en quelque sorte neutralisé le paramètre de la variation syntaxique, en ne considérant que l'une des constructions et en cherchant à caractériser filer dans cette construction-là.

En l'occurrence, et pour ce qui concerne ce verbe particulier, on choisit la construction intransitive ${ }^{8}:$ d'une part c'est l'une des constructions qui est compatible avec le plus de valeurs (comparer avec la structure attributive, toujours attributive), et donc où la variation du mot est la plus visible; d'autre part, la structure intransitive étant celle qui mobilise le moins de positions syntaxiques (du moins lorsque ce n'est pas une construction absolue), il y a un sens à considérer qu'elle pourrait être moins complexe, ou moins «construite», que les autres; du moins peut-on estimer qu'elle est celle qui fait intervenir le moins d'éléments extérieurs à filer.

\section{Reconstitution d'une forme schématique}

\subsection{Reconstitution 1 : le filant et son fil}

Soit la construction intransitive. Il s'agit de reconstituer ce qui serait la «fonction intégrative» attachée à filer dans l'ensemble des emplois que cette construction recouvre, par delà la diversité des co-textes lexicaux impliqués, et donc ici par delà la diversité des items lexicaux susceptibles d'intervenir en position sujet. Pour ce faire, on doit pour chaque emploi identifier les différentes entités mobilisées, puis déterminer ce qui est dit des entités en question. Cela suppose que l'on ait explicité la valeur que prend le verbe dans chaque cas, à l'aide de gloses précisant à chaque fois les ingrédients convoqués et la façon dont ces ingrédients sont configurés.

\footnotetext{
${ }^{8}$ Noter que ce pari prend le contrepied de ce que j'ai appelé l'option «propre », qui sélectionne plutôt un emploi transitif, celui où quelqu'un file quelque textile, pour donner la clef de ce que dit le mot. De fait, il ne parait pas possible dans tous les emplois de filer, de reconstituer quelqu'un filant quelque chose (dans le bas qui file, ou dans Pierre qui file au loin, qui file quoi ?), alors qu'il y a toujours quelque chose qui file, quel que soit la nature du « filage » en question.
} 
On cherche ce faisant à reconstituer ce qui serait la figure commune à l'ensemble de ces valeurs.

D'un emploi à l'autre, les gloses font apparaitre que l'on a affaire non pas à un mais à deux ingrédients, quelle que soit la valeur prise par le verbe et quel que soit le co-texte impliqué. Il y a ce qui file, qui peut être Mesrine ou le bas ou le caramel, mais qui en tout les cas correspondra toujours ici au référent du sujet: dans ce qui suit on note $\mathbf{y}$ cet élément. Mais il y a aussi ce qui tient lieu de fil, qui s'entend comme le produit du procès de filer, que ce fil soit un objet tangible (les fils du caramel par exemple) ou non (la fuite réussie de Mesrine qui doit être d'une façon ou d'une autre comme un fil, ou plutôt qui est donnée par l'expression comme étant de l'ordre du fil à certains égards) : on note $\mathbf{x}$ ce second élément. On voit que $\mathbf{x}$ ne correspond pas sur le plan syntaxique à un argument du verbe, mais figure plutôt ce que l'on appelle traditionnellement l'objet interne du verbe.

Les deux éléments sont très précisément configurés : $\mathbf{x}$ doit pouvoir tenir lieu de fil ; y doit être une entité susceptible de pouvoir filer.

Qu'y a-t-il de commun entre le gruyère, le caramel, le bas, le train, la rivière, Mesrine, l'argent ou le temps? Que dit le fait qu'ils soient susceptibles de pouvoir filer? On voit que tous, quoique chacun à sa façon sans doute, ont quelque chose à voir avec du fil justement : ainsi le caramel est-il constitué par le fait qu'il soit obtenu au terme d'une opération où un mélange est devenu filant ; le bas est fait de fil, et c'est bien parce qu'il est fait de fil, et même d'un seul fil, qu'il peut filer ; d'un autre point de vue, le temps, en tant qu'il est linéaire, unidimensionnel, et orienté, présente une structure qui n'est sans doute pas directement celle d'un fil, mais qui se manifeste comme telle; et le train, comme l'argent, circulent, sans reste, ce qui les rapporte à nouveau à une structure linéaire. Quant à Mesrine, il faut d'abord qu'il soit pris dans un mouvement linéaire pour que l'on puisse dire qu'il a filé : il faut qu'il se soit déplacé, et que l'on ait tenté de l'arrêter, d'interrompre son avancée. C'est dire que tous ces $\mathbf{y}$ voient sinon leur être même, du moins leur mode d'être dans l'occasion considérée, constitué par une forme de linéarité continue susceptible de se manifester à la manière d'un fil. C'est dire par conséquent qu'il faut que $\mathbf{x}$ soit la manifestation de ce qui dans le mode d'être de $\mathbf{y}$ était déjà de l'ordre du fil.

Quant à $\mathbf{x}$, il doit non seulement présenter les caractères formels de ce que l'on peut appeler un fil, par exemple ce que l'on a décrit ci-dessus en termes de linéarité et sur quoi on reviendra, mais il se trouve aussi placé dans une relation nécessaire avec $\mathbf{y}$. Les fils mis en place par filer ne sont pas seulement des entités linéaires plus ou moins tangibles et suffisamment continues : ils doivent tout autant, pour participer au schéma que met en place filer, être des « fils de » $\mathbf{y}$, c'est-à-dire à la fois être produits par $\mathbf{y}$ et être constitutifs sinon de $\mathbf{y}$ du moins de son mode d'être. Par exemple, les fils du caramel, ou plutôt le caractère filant du liquide qui doit se transformer en caramel, sont ce qui garantit qu'on a affaire à du caramel : en ce sens ils sont constitutifs du caramel. De même, l'échappée du fugitif n'est pas seulement ce qu'il advient de ce fugitif, c'est aussi comme on l'a vu ce qui fonde son mode d'être dans l'histoire que filer décrit: soit donc le fait que ce soit un fugitif, avant même qu'il ait pu effectivement filer. 
On a par conséquent affaire à un schéma dans lequel les deux pôles mis en place sont totalement noués l'un avec l'autre, dans une interrelation qui est circulaire : $\mathbf{x}$ se définit par $\mathbf{y}$ qui se définit par $\mathbf{x}$.

\subsection{Effets de syntaxe : entre filer et fil, la même figure du filant et $d u$ fil}

Ce rattachement des fils $\mathbf{x}$ à un $\mathbf{y}$ dont ils manifestent ce que l'on a appelé cidessus le «mode d'être» n'est pas simplement le propre des fils que convoque le verbe filer. On trouve un rattachement de ce type y compris avec le nom fil.

Ce nom fait lui-même l'objet d'une variation importante (du fil à coudre au fil de l'eau, en passant par le fil à pêche, le fil du rasoir et le fil de l'histoire), mais on peut considérer qu'il convoque dans chacun de ses emplois à la fois un $\mathbf{x}$ et un $\mathbf{y}$. Comme pour filer, $\mathbf{x}$ est alors sinon un fil du moins quelque entité plus ou moins tangible susceptible d'être de quelque façon rapportée à la notion de fil : par exemple dans le fil de l'épée, il est question de ce qui confère à l'épée son caractère effilé, et qui présente effectivement une linéarité sans épaisseur caractéristique des fils; dans le fil de l'eau, il est question d'eau courante, et en l'occurrence de ce qui détermine la direction du courant en question, une direction, qui comme celle du temps, partage avec les fils son caractère linéaire et ininterrompu. Et comme pour filer, ce fil se trouve rattaché à un autre terme $\mathbf{y}$ : celui-ci pourra être le référent d'un complément de nom, par exemple l'épée, l'eau, la viande dans les exemples cités, ou pourra être simplement implicite lorsqu'il n'y a pas de complément de nom. La différence avec filer est que $\mathbf{x}$ se voit cette fois nommé fil, tandis qu'en revanche $\mathbf{y}$ ne correspond plus nécessairement à un argument explicité du co-texte.

Dire que tout emploi de fil mobilise à la fois un $\mathbf{x}$ et un $\mathbf{y}$ revient à considérer ce mot comme étant fondamentalement un terme relationnel : tout « fil » est construit par le mot fil comme étant un fil de quelque chose.

De fait on observe un nombre important d'emplois de type prédicatif (le fil $d u$ temps, avec le temps qui est donné comme étant un fil ou comme étant constitué à la manière d'un fil) et un nombre non moins important d'emplois qui sont effectivement de type relationnel avec présence plus ou moins obligatoire d'un complément $\mathrm{C}$ (le fil du bois, de l'épée, du collier, de l'bistoire, etc.).

Et on vérifie que comme pour filer, il s'agit à chaque fois de dire que le fil en question est constitutif du y en question: constitutif de l'épée dans sa fonction, constitutif du courant qui rend l'eau « courante », mais aussi constitutif du bois dans sa substance propre, ou de l'histoire dans son déroulement, ou du fait que le collier tienne (puisque c'est lui, le fil $\mathbf{x}$, qui le retient, et qui fait de lui le collier qu'il est'). Dans le cas du fil électrique ou du fil de téléphone, comme aussi dans tous les cas où le nom est utilisé pour désigner des liens reliant divers objets ou divers points les uns aux autres (les fils qui nous rattachent les uns aux autres par exemple), y correspond aux liens en question: des liens de nature variée (physique, électrique, téléphonique ou autre) qui mettent en jeu une circulation (l'électricité, la voix du téléphone, le commerce

\footnotetext{
${ }^{9}$ Voir la formule de Flaubert que citent les dictionnaires : «Ce ne sont pas les perles qui font le collier, c'est le fil. »
} 
entre nous), et que les fils $\mathbf{x}$ tout à la fois rendent possible et matérialisent à leur manière discrète de fils.

L'analyse est sans doute plus délicate dans le cas du fil qui traîne, ou du fil à coudre surtout lorsque le nom, isolé, ne parait mobiliser aucun complément ${ }^{10}:$ Il me faudrait encore un peu de fil, J'ai acheté du fil, Cela a tenu à un fil, Ily a un fil qui dépasse, Ne tire pas sur le fil. Il y a cependant un sens à soutenir qu'un y est là aussi mobilisé. Selon les emplois ce $\mathbf{y}$ peut prendre des formes différentes : ce sera souvent la pièce (pièce de couture, habit) que le fil fait tenir (et qui donc a sa structure propre constituée par ce fil), pièce dont le rôle est de fait central dans la caractérisation de ce qu'est un fil lorsqu'il sert à coudre, puisqu'il est alors justement fait pour coudre et donc pour faire tenir ; ce sera aussi parfois l'ouvrage que le fil forme et constitue, dans le cas des fils du tissu ou des fils de broderie, le point important étant alors que ce tissu ou cette broderie, tout entiers faits de ce fil, tiennent tout entiers au fil en question, qui d'un seul tenant les constitue ; ce sera sans doute encore, dans d'autres contextes, non plus du tout ce que le fil sert à produire, mais ce dont il est tiré, à savoir par exemple la bobine, ou l'écheveau, entièrement faits de fil, et qui s'y dévident. L'idée générale est que les fils ne vont pas sans bobine, ou ne vont pas sans tissu, ou ne vont pas sans cousu, et que ce sera nécessairement l'un, l'autre ou le troisième que le mot fil va convoquer, y compris lorsque l'on parle simplement d'un «bout de fil qui traîne »: le fait même que l'on dise qu'il traîne est ici un indice de ce qu'il est mis en relation avec un tout auquel il se rapporterait, que ce tout soit bobine, tissu, ou ouvrage ${ }^{11}$.

Ainsi le lien entre $\mathbf{x}$ et $\mathbf{y}$, qui parait ici caractéristique de la figure du fil telle que la langue la construit au travers de ces expressions et de ces énoncés, entraîne-t-il subsidiairement une relation forte entre le nom fil et le verbe filer (du moins lorsque celui-ci est intransitif) : l'un et l'autre mettent fondamentalement en œuvre les mêmes éléments et les rattachent à la même configuration.

Dans cette mesure, il y a un sens à les rattacher à la même forme schématique, celle-ci décrivant la façon singulière dont une unité convoque un certain nombre d'entités pour les configurer.

On mesure ce qu'une telle hypothèse peut avoir de troublant : ce nom fil et ce verbe filer n'ont apparemment pas la même valeur, ne serait-ce que dans la mesure où le premier désignera plutôt une entité, tandis que le second désigne une forme de procès. Cependant le fait que filer désigne un procès n'est en aucun cas singulier : cela découle de l'appartenance du mot à la catégorie générale des verbes. Dès lors, cela n’a pas a priori à intervenir dans la forme schématique du mot, si l'on admet que la forme schématique se trouve exclusivement attachée à figurer ce que fait la singularité lexicale des unités.

On maintient par conséquent que filer et fil sont deux unités distinctes, mais on soutient que ce qui les distingue n'est pas singulier: on peut alors les rapporter à la même forme schématique, tout en associant chacun en outre à quelque schéma

\footnotetext{
${ }^{10} \mathrm{Il}$ est intéressant que ce soit à nouveau la valeur prototypique qui pose le plus de problème.

${ }^{11}$ On a aussi une difficulté avec le fil de pêche, mais on peut supposer que le fait que l'on parle là encore de fil renvoie à une sorte de théorie de la pêche, selon laquelle elle procède par lien (du pêcheur au pêché), ce lien étant matérialisé de manière aussi directe, linéaire et peu substantielle possible, par le fil en question, qui ne se voit pas, qui doit être droit, et qui lie.
} 
syntaxique caractéristique de la catégorie syntaxique dont il relève, à savoir une catégorie particulière de verbe d'une part, une catégorie particulière de nom d'autre part. On reviendra en 4.1. sur la forme que doivent prendre ces schémas syntaxiques. Le point important ici est qu'on est conduit à nouveau à distinguer plusieurs niveaux dans l'identité des mots : en l'occurrence un niveau notionnel, commun à filer et à fil, qui correspond à la forme schématique que la notion linguistique de fil met en œuvre, et un niveau syntaxique, où se différencient les catégories grammaticales. Cet étagement de l'identité lexicale ne coïncide pas exactement avec celui que l'on a déjà rencontré en 1. à propos de la variation des sous-catégorisations : il vient plutôt s'y ajouter, le niveau syntaxique qui rend compte du fonctionnement grammatical du mot (et qui oppose par exemple les noms et les verbes) étant distinct du niveau des structures distributionnelles de complémentation qui sont quant à elles variables (et qui opposeraient plutôt les transitifs et les intransitifs par exemple). On en arrive par conséquent à une identité étagée sur trois niveaux distincts. Cet étagement dit qu'au final filer et fil n'ont pas la même fonction intégrative, puisqu'ils n'ont pas les mêmes distributions, et puisqu'ils n'ont pas les mêmes arguments (un sujet dans un cas, un éventuel complément de nom dans l'autre). Ils ont cependant des fonctions intégratives qui peuvent être reliées l'une à l'autre parce qu'ils mobilisent la même forme schématique.

\subsection{Reconstitution 2 : le redoublement des fils}

Que ce soit pour fil ou pour filer, la notion de fil mobilise en fait deux fils, se redoublant l'un l'autre : d'une part le $\mathbf{x}$ que fil désigne et dont filer dit qu'il se manifeste (sous une forme plus ou moins tangible); d'autre part ce qui constituait le mode d'être de $\mathbf{y}$ avant même qu'on ait dit qu'il file, qui est déjà une forme de fil, et qui est en tous les cas ce que $\mathbf{x}$ en tant que fil manifeste, et que l'on propose de noter $\mathbf{X}$. Ce redoublement joue un rôle déterminant dans la façon dont se constitue la figure du fil : il explique le fait que les fils soient tout à la fois des entités séparées et les parties intégrantes d'un tout auquel elles s'ajustent et qu'elles constituent.

Cette dissociation entre le fil qu'est $\mathbf{x}$ et le caractère filable de $\mathbf{y}$ qu'est $\mathbf{X}$ est particulièrement nette dans le cas du fugitif qui a filé. On a d'un côté sa fuite réussie $\mathbf{x}$, que l'expression rapporte à la figure du fil, linéaire et ininterrompue, et on a d'un autre côté ce qui constituait son mode d'être avant qu'il réussisse, à savoir un mouvement $\mathbf{X}$ d'échappée, virtuellement linéaire et ininterrompue, mais qui n'était qu'échappée virtuelle précisément.

De même dans le bas qui file, on a du fil avant et après que le bas ait filé, mais les deux fils n'ont ni le même statut ni en l'occurrence la même forme ou la même matérialité : on a d'un côté le fil qui constituait le bas, qui correspond à $\mathbf{X}$, mais qui n'est pas directement ce que l'on voit se manifester quand le bas commence à filer; ce qui se manifeste, donc $\mathbf{x}$, est ici ce que l'on appelle aussi une échelle, qui est linéaire comme le fil $\mathbf{X}$ constituant le bas, qui donne à voir en outre ce fil $\mathbf{X}$, mais qui est verticale quand $\mathbf{X}$ s'enchaîne horizontalement (d'où sa forme en échelle, avec le $\mathbf{X}$ rendu apparent en barreaux de l'échelle). C'est justement parce que $\mathbf{X}$ est d'un seul tenant que l'échelle $\mathbf{x}$ va se trouver difficile à arrêter, et sera comme un fil, à la fois linéaire et a priori illimitée. 
De même encore, dans le fil de l'histoire, l'histoire est d'abord constituée par une forme d'enchaînement, qui lui confère a priori son caractère linéaire, et qui correspond à $\mathbf{X}$; cet enchaînement va se manifester lorsque l'histoire est racontée, entendue ou lue ou vécue, comme une continuité qu'il ne s'agit pas d'interrompre et qui donc se donne comme un fil $\mathbf{x}$ que l'on peut alors perdre, suivre, reprendre, qui serait plutôt le fil du récit, ou le fil de la lecture, que celui de l'histoire racontée elle-même.

Dans le fil du collier, ou dans le fil du téléphone, il y a d'abord le collier, et le téléphone qui en eux-mêmes mettent en jeu une linéarité $\mathbf{X}$, celle de l'enchaînement des perles, celle du lien qui se réalise entre les personnes qui communiquent par téléphone; et il y a ensuite le fil $\mathbf{x}$ qui est comme la matérialisation, et aussi la garantie ou la réalisation, du lien $\mathbf{X}$ constitutif de $\mathbf{y}$.

Dans le fil de la bobine, et le fil du tissu, la dissociation des deux formes de fil, $\mathbf{x}$ et $\mathbf{X}$, prend une autre dimension: $\mathbf{x}$ est alors le fil isolé et autonome, pris dans sa longueur, plus ou moins homogènement filé, qui ne sait plus grand-chose en tous les cas de la fibre d'où il vient; $\mathbf{X}$ est le fil enroulé, tissé ou cousu, ajusté au $\mathbf{y}$ auquel il se destine, dont la linéarité a pris une autre forme, celle de l'enroulement indéfini, du tissage structuré, ou du dessin linéaire que font les points de couture parcourant la pièce cousue, et dont la continuité correspond au fait qu'il soit d'un seul tenant, sur la bobine, dans la trame du tissu, ou tenant la pièce ${ }^{12}$. Et c'est parce qu'il y a cet écart entre $\mathbf{x}$ et $\mathbf{X}$ qu'il faut que le fil soit souple, propre à s'ajuster à quelque $\mathbf{y}$ que ce soit.

Pour prendre un dernier cas, dans le fil d'Ariane, où $\mathbf{y}$ est sans doute la figure même du labyrinthe, $\mathbf{X}$ correspond au chemin à suivre dans ce labyrinthe (constitutif de la façon dont le labyrinthe est ce qu'il est), et $\mathbf{x}$ est le fil, qui va très exactement venir s'appliquer sur $\mathbf{X}$, pour le redoubler, et le marquer ensuite comme chemin à suivre vers la sortie ${ }^{13}$.

\footnotetext{
${ }^{12}$ La configuration n'est pas très différente pour le fil d'baricot. On a là aussi sinon deux fils, du moins deux fois le même fil, mais les deux fois avec des formes et des statuts bien différents : il y a le fil séparé du haricot, une fois retiré, qui tend à se recroqueviller sur le papier journal où il traîne avec ses congénères; et il y a le même fil encore adhérent au haricot, dont on verra l'empreinte. Là encore, il est constitutif du fil de haricot qu'il y ait ces deux états du fil, que le fil soit à la fois ce qui traîne, et est manifeste, et ce qui ne se voit pas et adhère au haricot.

13 Pour le fil du bois, la dissociation entre $\mathbf{x}$ et $\mathbf{X}$ est sans doute plus difficile à justifier. Reste que $\mathbf{x}$ correspond là aux fils qui «se voient», se dessinent le long du bois, ou se perçoivent au toucher. Or le fil du bois ne consiste pas simplement dans les quelques fils qui s'y dessinent. S'il y a fil du bois, c'est dans la mesure où tout le bois est fil, y compris quand ce caractère filandreux ne se matérialise pas à la vision ou au toucher. $\mathbf{X}$ serait donc ici l'ensemble des fibres du bois, dont les fils $\mathbf{x}$ ne sont que la manifestation perceptible. Pour le fil de la viande, la situation est encore plus difficile a priori, dans la mesure où ces fils ne se voient pas. Mais ils se perçoivent au couper : $\mathbf{x}$ est donc ce sur quoi la coupe glisse, ce que l'on perçoit à la coupe, tandis que $\mathbf{X}$ est à nouveau la propriété générale de cette viande d'être constituée de fibres longues.
} 


\subsection{Petit détour du côté des réalités : ce que sait le mot fil de ce que c'est qu'un fil}

En mettant en scène un tel redoublement entre un fil constitué et manifesté d'une part, un fil constituant et virtuel d'autre part, la notion de « fil » dit sans doute quelque chose d'essentiel sur toutes ces choses, tangibles ou non, que les mots fil et filer désignent. En particulier, elle capte plutôt élégamment ce que peut être l'objet concret que l'on appelle fil de manière prototypique : ce fil-là, le fil «textile », se trouve en effet être pris quant à lui dans deux redoublements, puisqu'on l'obtient à partir d'une fibre textile, donc d'une matière se trouvant elle-même constituée de fibres filables, et que l'on file de manière à ce qu'elle puisse être ensuite tissable, ou utilisable pour coudre ou broder. Il n'y a pas de fil textile qui ne soit obtenu sans fil préalable (les fibres), et qui n'ait pour fonction possible de tenir lieu de fil pour un tissu (ou une pièce cousue ou une broderie). Tel est le nœud technologique que dit d'ailleurs le mot textile lui-même, et qui se trouve en fait inscrit dans l'étymologie de ce dernier : le «textile» est du «qui peut être transformé en «texte»», donc en «tissu », et se définit de fait comme étant une matière constituée de fibres dont on peut faire des fils que l'on peut alors tisser.

Ces liens entre fil, fibre, textile et tissu sont sans doute constitutifs de la façon dont chacune de ces entités réelles se trouve conceptualisée, y compris indépendamment de toute verbalisation, à travers par exemple les pratiques qu'elles mobilisent (couture, broderie, enfilage d'aiguille, tissage, etc.), ou à travers les caractérisations scientifiques qui pourraient en être données (caractérisation de ce qu'est une fibre, de ce qu'est un textile par exemple). Dans la mesure en outre où deux d'entre elles sont directement des objets technologiques (qui n'existent pas sans une technologie), il est clair qu'elles sont, dans leur réalités même, cognitivement construites. Les mots fil et filer, en mobilisant $\mathbf{x}, \mathbf{X}$ et $\mathbf{y}$, rencontrent ces conceptualisations diverses. Ils les rencontrent, mais ils ne se contentent pas de les refléter. Car il n'y a pas une seule conceptualisation que chaque medium retracerait. Il y a plusieurs conceptualisations pour une seule réalité, et ces conceptualisations ne font que se rencontrer, elles ne se reproduisent pas : le fil de la couturière, n'est pas le fil de la brodeuse, n'est pas le fil de la scientifique, n'est pas le fil qui dépasse du tailleur de la ministre ; et aucun de ces fils n'est la figure que construit le mot fil, aussi informé celui-ci soit-il de ce qui travaille toutes ces conceptualisations, et qui est la réalité technologique du fil. Si la conceptualisation que dit le mot n'était que le reflet d'un construit cognitif extérieur, le mot dirait toute la chaîne technologique qui relie le textile au tissu via le filage puis le tissage du fil ; et il dirait aussi la broderie, la couture, l'enfilage des aiguilles, les fils qui dépassent, et tout ce qui fait notre vécu des fils. La notion extralinguistique de fil textile est sans doute une notion complexe et construite, mais elle est justement trop complexe pour ce que la langue en dit: la langue ne dit qu'un redoublement, celui de $\mathbf{x}$ et $\mathbf{X}$, et non pas le redoublement entre fil et fibre d'une part, entre fil et trame d'autre part; elle ne dit qu'une relation, la relation de $\mathbf{x}$ à $\mathbf{y}$, et non pas à la fois la relation du fil à la matière textile et la relation du fil au tissu. On mesure ainsi le lien qui relie la notion linguistique de fil et ses référents. Il s'avère que cette notion linguistique est bien une figuration de ces référents, qu'elle est en tant 
que telle informée de ce qui fait la complexité tant cognitive que réelle des référents en question (et on verra d'ailleurs qu'elle n'est pas informée que du seul redoublement, qu'elle sait d'autres choses décisives, qu'elle dit d'autres choses décisives sur ce qui fait d'un fil un fil), mais qu'elle n'est pas pour autant le simple reflet de la notion technique de fil textile (elle sait que le fil suppose la fibre, et que le fil est fait pour faire du tissu), ou de la technologie du filage (avec quenouille, métier à tisser, et les gestes définis qui s'y rapportent), telles que construites cognitivement. C'est l'analyse linguistique de la valeur des mots d'un emploi à l'autre, et non l'analyse cognitive du mode de construction de leur référent, qui permet de la reconstituer. Dans cette mesure c'est bien d'une notion linguistique qu'il s'agit: elle n'est "physico-culturelle» selon l'expression un peu lourde adoptée par les culioliens, qu'en tant qu'elle est la façon dont la langue saisit le «physico-culturel », et non pas en tant qu'elle serait déterminée extérieurement aux langues.

\subsection{Reconstitution 3 : le filant du filant}

Si l'on en croit les gloses qui ont permis d'expliciter les valeurs obtenues dans les différents emplois observés, le réseau mis en place par la notion linguistique de « fil » comporte au moins une position en plus des trois déjà mises en évidence. En effet, indépendamment de ce que l'on a décrit comme un redoublement du fil, il faut aussi que la position correspondant à l'entité qui est supposée filant soit dédoublée : car ce n'est pas directement $\mathbf{y}$, mais ce que l'on a appelé son "mode d'être », que l'on notera donc $\mathbf{Y}$, qui se voit mis en relation avec le fil que désignent $\mathbf{x}$ et $\mathbf{X}$.

En parlant de "mode d'être », on vise dans la plupart des cas ce qui prend l'allure d'un état transitoire de $\mathbf{y}$, ou d'une façon d'être: par exemple dans le cas du fromage, ou de la maille, mais aussi de l'oiseau, qui les uns et les autres ne sont pas toujours filant, et qui en eux-mêmes n'ont rien de spécialement linéaire ou continu pouvant être rapporté à une forme de fil ; c'est seulement dans un certain état, lorsqu'ils fondent, ou se défont, ou volent à tire d'aile, que gruyère, maille ou oiseau ont à voir avec du fil ; et même dans cet état, ce n'est pas tant eux que l'état lui-même qui peut être rapporté à du fil.

Sans doute y a-t-il des cas où le lien entre $\mathbf{y}$ et la forme fil parait plus serré : par exemple dans le cas du fugitif ou dans celui du caramel où l'on a vu que le fait de filer est ce qui fonde l'un comme l'autre dans leur être respectif de fugitif ou de caramel. Mais même dans ce cas, ce n'est pas le fugitif ou le caramel qui en eux-mêmes sont du fil, c'est seulement ce qui fait d'eux des fugitifs et du caramel, à savoir la fugue de l'un et le caractère filant de l'autre. C'est donc bien à nouveau leur «mode d'être », quoique l'expression désigne là quelque chose de moins transitoire et qui est plutôt de l'ordre de la condition d'existence.

Il est des cas enfin où l'on peut considérer que $\mathbf{y}$ lui-même a la forme d'un fil : ainsi en est-il du temps ou du chemin, qui l'un et l'autre sont intrinsèquement linéaires et suffisamment continus pour pouvoir être décrits comme tels. Reste que même alors, en prédiquant du temps ou d'un chemin qu'ils filent, on ne se contente pas de décrire leurs formes respectives: on rapporte une situation, dans laquelle on a le sentiment que le temps passe très vite, ou on décrit un paysage dans lequel le chemin est perçu comme formant un fil (sans doute parce qu'il atteint en droite ligne, et donc 
sans s'attarder, un point où il disparaît de la vue). On voit ainsi que ce n'est en fait pas leur forme elle-même qui est en jeu, mais bien la façon dont cette forme se manifeste dans une situation donnée : soit à nouveau ce que l'on peut appeler son mode d'être dans la situation en question.

A chaque fois par conséquent, quoique de manière différente, c'est le mode d'être $\mathbf{Y}$ qui figure la part de filant dans $\mathbf{y}$.

Par ailleurs, le mode d'être en question ne coïncide pas pour autant avec $\mathbf{X}$ : ils sont clairement distincts dans le cas par exemple du gruyère où $\mathbf{Y}$ correspond à l'état du gruyère quand il est fondu, et où $\mathbf{X}$ correspond aux fils potentiels qui sont constitutifs de cet état. $\mathbf{X}$ est bien ce qui « constitue » $\mathbf{Y}$, et il faut à nouveau entendre cette relation dans tous les sens qu'elle peut avoir, suivant que le lien qu'elle pose entre $\mathbf{X}$ et $\mathbf{Y}$ est plus ou moins serré : elle peut signifier selon les cas que $\mathbf{X}$ est un simple constituant de $\mathbf{Y}$, ou qu'il est un élément constitutif de $\mathbf{Y}$, voire même qu'il est ce qu'est $\mathbf{Y}$. On verra en 3. que tous les cas de figure sont représentés. Cette variation même suppose par conséquent que $\mathbf{X}$ et $\mathbf{Y}$ soient dissociés.

On en arrive dès lors à une configuration qui articule en définitive quatre positions. La relation fondamentale qui rattache $\mathbf{x}$ à $\mathbf{y}$ s'avère être la composée de trois relations : $\mathbf{x}$ est la manifestation de $\mathbf{X} ; \mathbf{X}$ constitue $\mathbf{Y} ; \mathbf{Y}$ est le mode d'être de $\mathbf{y}$. On reviendra ci-dessous sur ce qui fait le détail de ces rattachements, et sur la façon dont ils s'élaborent: il va s'avérer que chaque lien est tout à la fois une source de signification (dans la notion de fil, il est question de manifestation, et il est question de constitution, et il est question de mode d'être) et une occasion de variation, aucun ne pouvant dès lors être escamoté dans la caractérisation que l'on cherche à établir de ce que décrivent les lexèmes fil et filer.

\subsection{Reconstitution 4 : la forme des fils, ou ce qui fait que les fils sont des fils}

Le rattachement de $\mathbf{x}$ à $\mathbf{y}$ d'une part, la dissociation entre $\mathbf{x}$ et $\mathbf{X}$ d'autre part, le dédoublement de $\mathbf{y}$ et $\mathbf{Y}$ enfin, tout cela atteste d'une forme de fragmentation de ce que peut mettre en scène la notion linguistique de «fil». Reste qu'en mettant en évidence cette fragmentation, on n'a toujours pas expliqué véritablement en quoi consiste le fait d'être du fil: on a simplement déplacé le problème en montrant comme la notion se distribuait sur le réseau des entités référentielles qu'elle mobilise. Si la forme schématique que l'on cherche à reconstituer doit décrire ce qui fait la singularité de cette notion, elle doit comporter, outre la configuration à laquelle on a abouti, une explicitation de ce qui unit toutes les entités mobilisées, à savoir le fait qu'elles aient les unes et les autres à voir, plus ou moins directement, avec du fil. Il s'agit en l'occurrence de comprendre ce que recouvrent les deux propriétés que l'on a évoquées à plusieurs reprises, en parlant de linéarité d'une part, de continuité d'autre part, l'idée étant que ces deux propriétés conjuguées suffisent à caractériser la forme qui doit être celle d'un fil et qu'exprime de fait la notion de fil.

\subsubsection{Linéarité : le sens d'un parcours}

On va soutenir que ce qui est en jeu dans le fait que $\mathbf{x}$ comme $\mathbf{X}$ doivent être linéaires est bien plus qu'une affaire de forme: donner $\mathbf{x}$ et $\mathbf{X}$ comme étant des 
structures linéaires revient à les donner comme constituant de véritables parcours, sur lesquels peut alors se déployer un mouvement, au moins virtuel.

Que la notion de linéarité doive s'entendre en terme de parcours peut sembler paradoxal ${ }^{14}$. On comprend mieux le sens que cela peut avoir si l'on réfléchit à ce que met en jeu le concept de parcours. A nouveau il s'agit d'un concept ambigu, et à nouveau cette ambiguité est à prendre comme un symptôme de ce que le concept recouvre : selon les cas, le parcours correspond ou bien à un déplacement, ou bien au chemin auquel ce déplacement se conforme. S’il y a une telle ambiguité, c'est bien parce que cette conformité entre un déplacement et un chemin se régulant mutuellement est très exactement ce que dit le concept : un parcours est un chemin propre à réguler un déplacement, ou bien est ce déplacement lui-même en tant qu'il détermine un chemin à suivre. Or, derrière cette idée de corégulation entre chemin et déplacement, c'est la problématique de l'alignement et donc de la linéarité qui se profilent : chemin et déplacement sont alignés l'un sur l'autre. Une structure linéaire est une structure propre à définir la ligne sur laquelle un déplacement devra s'aligner : c'est donc une structure propre à faire fonction de parcours ${ }^{15}$.

De fait, dans l'usage courant qui est fait de cette notion, une structure linéaire est une structure unidimensionnelle. C'est dire qu'elle est dépourvue d'épaisseur, discrète, aussi peu matérielle que possible. Mais cela va aussi avec l'idée qu'elle définit un parcours obligé : à la différence de ce qui se passe dans une surface ou un volume, où l'on a le choix entre plusieurs chemins, il y a ici un seul chemin possible. L'unidimensionnalité s'entend donc comme une contrainte sur un cheminement. D'autant qu'en l'occurrence, avec la linéarité, l'unidimensionnalité est une affaire non pas de hauteur ou de largeur mais de longueur : la structure linéaire est une structure dont toute la dimension est dans la longueur (une structure «allongée et fine » dit le Petit Larousse). Or, la longueur elle-même est une notion difficile, qu'il faudrait examiner en propre, mais qui semble être fondamentalement un problème de distance : on est toujours inscrit dans le registre du déplacement, la longueur mesurant la distance à parcourir d'une extrémité à l'autre ${ }^{16}$.

\footnotetext{
14 On ne s'intéresse pas au mot linéarité, mais bien à la notion, extralinguistique, que ce mot peut nommer. De même pour les autres notions qui vont être évoquées dans ce qui suit : notion de parcours, de dimension, de longueur, de continuité. Ce sont les notions dont on cherche à prendre la mesure, pour comprendre ce qui peut caractériser la forme fil. Pour ce faire, il n'est pas impossible que l'on tienne compte du sens que peuvent prendre les mots linéarité ou parcours ou autres, dans la mesure où l'on a vu qu'il y avait de fait un rapport entre le sens des mots et les entités extralinguistiques que ces mots désignent: les mots sont informés de ce qui constitue ces entités qu'ils se trouvent de fait affectés à dire. Le mot linéarité peut donc nous dire des choses sur ce qu'est la linéarité, des choses qui seront éventuellement des choses utiles pour comprendre ce que cette propriété peut mettre en jeu. Reste que c'est là la propriété qui nous intéresse dans ce qu'elle est et non dans la façon dont le mot la donne.

${ }^{15}$ La notion mathématique de linéarité est fondée sur une telle proportionnalité, avec deux variables dont l'une est reliée à l'autre par la formule $\mathrm{y}=\mathrm{ax}$.

16 Que la longueur soit affaire de parcours explique par ailleurs que le terme soit appliqué à la fois pour mesurer l'espace, et plus spécifiquement donc les distances à parcourir, et pour mesurer le temps ("Ce fut long»).
} 
Quoi qu'il en soit, on va voir que c'est bien la catégorie du parcours que la notion linguistique de «fil » met en scène et que cela constitue une caractéristique inattendue mais néanmoins incontournable de tous les emplois que l'on peut faire des mots fil et filer: à chaque fois, il est question d'un mouvement au moins virtuel, et à chaque fois $\mathbf{x}$ ou $\mathbf{X}$ figurent la ligne sur laquelle ce mouvement doit s'aligner. Les fils qu'évoquent ces mots s'avèrent dès lors être bien plus que des entités plus ou moins tangibles dont la forme serait tout à la fois aussi peu épaisse que possible et exclusivement allongée : ce sont des parcours virtuels pour quelque objet parcourant.

Cela s'entend par exemple dans le fil de l'bistoire, qui mobilise nécessairement un récit, une écoute, un film ou quelque forme que ce soit de déroulement de l'histoire en question, et, à travers ces déroulements, un sujet qui raconte, écoute, visionne ou assiste. Cela s'entend aussi dans le fil du bois, que l'on peut suivre avec le doigt et qu'il vaut mieux suivre quand on le débite en planches ou en petit bois : ce fil du bois évoque donc le doigt qui l'explorera, ou la découpe qui s'y conformera ; comme le fil de l'eau évoque le mobile qui se laisserait porter par son courant. Cela s'entend, d'une autre façon, dans le fil d'Ariane, qui est bien un fil à suivre, pour quelque Thésée que la quête a mis en mouvement. Cela s'entend aussi bien dans les fils électriques et autres fils de téléphone où l'on a déjà noté une nécessaire circulation, d'électricité ou de signaux. C'est sans doute ce qui explique que le fil des couturiers, brodeurs, tisserands et des fileurs toujours évoque quelque geste virtuel, qui l'effile, le déroule, l'enfile ou l'allonge. Et c'est ce qui se manifeste explicitement dans toutes les valeurs de filer qui impliquent un mouvement ou un changement de $\mathbf{y}$. Mais c'est alors y lui-même qui figure l'objet parcourant, et son mode d'être est ce mouvement : dans le navire filait, on a $\mathbf{y}$ qui est le navire, son mouvement qui est son mode d'être, qui est déterminé par sa direction $\mathbf{X}$ se manifestant sous la forme d'un fil $\mathbf{x}$. De même dans l'argent qui a filé, il faut expliquer que c'est en étant dépensé que l'argent a ainsi filé : or, qu'il y ait en jeu une affaire de dépense se déduit du fait que l'argent est donné comme support d'un parcours ; car c'est bien en étant dépensé que pour sa part l'argent circule. Y compris dans le caramel ou le gruyère filant, il s'agit de dire l'apparition progressive des fils, et non pas simplement leur existence : c'est quand j'extrais une nouille du plat que le gruyère file, dans ce mouvement d'étirement auquel il est soumis ; c'est quand je remue, que les fils de caramel se dessinent, et non pas spontanément. On retrouve donc là l'idée d'un mouvement qui vient coïncider avec les fils apparus. Dans la lampe qui file, le fil renvoie sans doute à l'apparition d'une ligne de fumée au bout d'une flamme qui par ailleurs s'effile, mais ces lignes perceptibles - celle de la fumée, prolongeant celle que fait la flamme - sont nécessairement attachées à un déroulement, celui de la lampe qui brûle, et à un mouvement, celui de cette flamme et de cette fumée s'étirant verticalement vers le haut. Dans le fil du rasoir, le mouvement est un geste, celui que l'on fait quand on use de ce fil-là, avec la lame perpendiculaire à la peau, «sur le fil », et la peau qui s'ouvre dans ce mouvement glissant à sa surface. Le fil de l'épée est de ce point de vue particulièrement éclairant, parce qu'il devrait simplement désigner une partie de l'épée, son fil, par opposition à sa pointe, alors que l'expression ne s'emploie là encore qu'en relation à un geste, qui consiste à avoir enfoncé l'épée dans son long: c'est qu'à nouveau le fil dit un mouvement, dont il fournit en outre la direction - au travers du corps visé. 
Dans tous ces cas donc il y a mouvement, y compris là où l'on ne s'y attend pas, notamment lorsqu'il ne s'agit pas du verbe mais du nom, y compris aussi quand le mouvement prend la forme d'un déroulement temporel, y compris surtout lorsque ce mouvement n'est que le geste virtuel que le fil peut appeler (geste du brodeur, de l'éplucheur d'haricots, de l'enfileur de perles, de la hache sur le bois, mouvement de la coque de noix à la surface de l'eau, et même, ainsi qu'on le verra, geste du pêcheur qui déroule sa ligne ou qui l'étire d'un mouvement de canne à pêche). L'idée est qu'un tel mouvement serait absolument nécessaire à ce que l'on puisse convoquer la notion de fil pour désigner quelque objet ou quelque entité que ce soit.

La difficulté est que la façon dont ce mouvement se greffe sur les différentes entités mises en œuvre par la notion est variable. Tous les cas de figure semblent en fait envisageables : ce peut être $\mathbf{x}$ lui-même qui figure ce mouvement (ce serait le cas avec le train file où c'est bien le mouvement du train qui est décrit comme ayant la forme d'un fil), mais $\mathbf{x}$ peut n'en être que le produit (dans le cas du bas ou du gruyère), ou bien peut en être l'objet (ce serait le cas pour le fil à coudre), ou enfin le support (dans le cas du collier, où le fil est ce sur quoi les perles ont été enfilées); ce peut être déjà au niveau de $\mathbf{X}$ qu'il y a mouvement (ce serait le cas du temps, qui renvoie de manière intrinsèque à un déroulement, indépendamment de ce qui peut manifester quand il apparait que «le temps file »), et ce peut aussi être $\mathbf{X}$ qui en figure le produit, l'objet ou le support. De toutes façons, les mots fil et filer ne disent pas en eux-mêmes qu'il y a mouvement, sauf dans les quelques cas qu'on a vu où filer est verbe de mouvement. Simplement, et de manière variable, ces mots supposent un mouvement de ce type, qui vient en quelque sorte ourler, fonder, donner sa consistance à la figure dessinée par la notion.

Le fait que le mode de rattachement du mouvement soit variable est clairement une des sources importantes de la variation à laquelle donnent lieu aussi bien fil que filer. C'est aussi l'origine du travail interprétatif important que ces mots peuvent déclencher: il faut dans les mots du co-texte, ou à défaut quelque part dans le contexte, reconstituer ce qui pourrait de quelque manière définir un parcours, pour quelque élément en mouvement ${ }^{17}$.

17 On explique ainsi ce qui peut poser problème dans l'exemple suivant pourtant attesté : « deux grands arbres entre lesquels un jeune tilleul filait ». Dans ce cas, le fil parait n'être qu'affaire de forme, et on peine à imaginer ce tilleul, à l'envisager le plus mince possible, ou au moins émincé, avec un feuillage qui part en pointe, telle la fumée de la bougie qui file. Pourtant, on sent bien que ce n'est pas exactement cela qui est en cause : le tilleul en question doit sans doute être assez peu épais, assez allongé, et surtout assez fragile, mais on n'est pas sûr qu'il doive véritablement faire figure de fil. Le problème, et la difficulté, est plutôt qu'il soit pris dans un mouvement: on bute sur le fait qu'il faudrait qu'il soit soumis à un mouvement, et on ne voit pas en quoi ce mouvement pourrait consister. C'est là sans doute qu'intervient la donnée des autres arbres, qui déterminent la ligne que celui-ci peut suivre. C'est là peut-être qu'on sera aussi tenté de se rabattre sur la jeunesse de l'arbre, et sur le fait qu'il pourrait pousser ... filant alors vers le haut. L'exemple est difficile à interpréter parce qu'on ne sait pas sur quoi va pouvoir se greffer le mouvement. 


\subsubsection{Continuité : le point de non-retenue}

On a un parcours, et filer dit qu'il est ininterrompu, ou fil dit qu'il est d'un seul tenant; dans les deux cas le parcours est présenté comme étant sans rupture, donc continu. La linéarité est déjà en elle-même une forme de continuité, avec un mouvement qui se déploie continûment sur le parcours qui le détermine. L'élément supplémentaire ici est d'une certaine façon négatif : dans ce parcours, il n'y a pas de point d'interruption ${ }^{18}$. Cela revient à dire que la continuité est totale, d'un bout à l'autre du parcours. Plus précisément, il s'agit de poser que là où le mouvement aurait pu s'interrompre, il ne s'interrompt pas. Dans tous les cas en effet, on va montrer que derrière ce que l'on cherche à décrire en termes de continuité, il y a d'abord la mise en place d'une sorte de point fixe; ce point est alors donné comme un point où le mouvement continue : un point de continuité donc, ou plutôt un point de «nonretenue».

A nouveau le point en question va pouvoir prendre différentes formes, et va pouvoir s'accrocher de différentes façons au parcours par rapport auquel il intervient. Ce peut être le spectateur, qui observe le paysage, dans le cas des rivières, chemins, et autres oiseaux: l'idée est alors que les rivières, chemins et oiseaux en question s'éloignent au plus vite du spectateur, qui ne les a pas retenus. Dans le fil de l'bistoire, le point fixe est l'auditeur qui n'interrompt pas; c'est le passant qui voit passer impuissant le train qui file et ne peut que le suivre des yeux; c'est l'inspecteur qui cherchait à arrêter le fugitif quand il a malgré tout filé : dans tous ces cas, il s'agit en fait d'un point d'interruption virtuelle; et ce point virtuel, la notion de « fil » semble avoir suffi à le convoquer. Dans le cas de l'argent, de la vie, ou du temps, le point fixe est le locuteur, pour qui cet argent, cette vie, et ce temps filent, et qui de fait dit là ne pas réussir à les retenir ou à les conserver: ce paramètre du point de non-retenue permet à la fois de rendre compte du caractère subjectif de l'assertion, et d'expliquer que le filage s'interprète en termes de perte et de disparition. Dans le cas du fil de l'eau, le point fixe est la coquille de noix ou autre embarcation qui se laissent dériver à la surface de cette eau, et qui doivent nécessairement être impliquées, au moins virtuellement, pour que l'on puisse parler en l'occurrence de fil: une eau qui coule, depuis le robinet jusqu'à la baignoire, ne détermine pas un «fil», aussi courante soitelle.

Cette affaire de point fixe est particulièrement précieuse pour expliquer tous les cas où le fil met en scène un étirement ou un déroulement: ainsi dans le cas du gruyère, où le point fixe est la nouille à laquelle le fil adhère, mais aussi dans le cas du câble qui file, où il est question d'un câble que l'on a lancé dans l'eau et qu'on laisse se dérouler tandis que le bateau avance, et encore dans le cas du fil de pêche, qui va de la même façon se tendre et se dérouler depuis le point où l'hameçon a été jeté. Pour les fils à coudre, le point fixe est selon les contextes l'amorce de la bobine, l'aiguille, ou le point que l'on vient de faire, à partir desquels le fil s'étire. On voit qu'à chaque fois le point fixe permet de rendre compte de la forme même du geste par lequel le fil se trouve étiré : un geste de séparation (dans le cas du fil de haricot, ou dans celui du fil

18 «On ne lève pas son crayon» comme le disent les enseignants qui initient l'apprenti mathématicien aux fondements de la continuité. 
de gruyère), ou un geste de déroulement (pour le câble, le fil de pêche, le fil de la bobine).

Dans le fil d'Ariane, le point fixe est sans doute le centre du labyrinthe où il s'agit que Thésée ne soit pas retenu, et à partir duquel s'organise tout le déroulement de la sortie que le fil doit permettre.

Un des effets les plus intéressants est celui qui s'attache aux cas où le fil est circulaire : parce que le point fixe est alors ce qui permet de dire cette circularité et les retours qu'elle implique. Ainsi dans le cas du collier, mais peut-être aussi dans le cas du téléphone: le point fixe est celui où la boucle retourne sur elle-même, où elle pourrait s'achever et où elle continue. Le cas du pouls n'est pas très éloigné, avec la cyclicité du rythme tenant lieu de boucle. Mais tout se passe alors comme si deux effets se cumulaient: le pouls qui file serait à la fois un pouls de plus en plus imperceptible, qui échappe donc à celui qui tente de le prendre (premier point fixe), et un pouls qui perd le rythme, parce que le point de retour où la pulsation devrait se clore (deuxième point fixe) n'arrive pas, et que la pulsation, ralentie, continue, et se perd.

Qu'il y ait un point de non-retenue permet ainsi à chaque fois d'expliciter dans le détail la figure que l'expression met en scène, le geste dont cette figure procède, la forme que ce geste peut prendre. C'est bien cela qui, ajouté au fait qu'il y ait parcours, parvient à donner leur substance aux différents effets de sens qui se trouvent attachés à chaque emploi ${ }^{19}$.

\subsection{Reconstitution 5 : des strates dans les formes}

On arrive au bout du travail de reconstitution, avec à la clef une caractérisation consistante de la configuration qui se trouve déployée par la notion linguistique de « fil ».

Trois éléments référentiels supplémentaires ont été introduits en 2.6. quand on a voulu expliciter le contenu qualitatif de cette notion: le mouvement, l'objet en mouvement et le point de non-retenue. Les deux premiers peuvent être simplement virtuels. Le troisième est nécessairement actualisé. Les uns et les autres ont par ailleurs la particularité de n'avoir cette fois rien à voir avec des fils, ou avec du filant ou avec du filable. Il s'agit véritablement de positions extérieures, à partir desquelles on a enfin pu déconstruire le concept de fil. On ne sait par ailleurs pas exactement la façon dont ils se rattachent aux autres éléments référentiels impliqués que sont $\mathbf{x}, \mathbf{X}, \mathbf{y}$ et $\mathbf{Y}$. On sait seulement que ces éléments sont nécessaires et qu'il faudra que l'interprétation fasse le travail de trouver comment ils peuvent se mettre en place à partir de tel ou tel co-texte et/ou dans tel ou tel contexte référentiel.

La forme schématique à laquelle on aboutit ménage ainsi deux strates dans la configuration qu'elle établit : l'une qui correspond à ce que la notion dit, où il est question de fils, de filables et de filants, pris dans certaines relations bien définies, avec en particulier les fils qui s'interprètent comme déterminant un parcours non

\footnotetext{
${ }^{19}$ L'idée du point de non-retenue avait été proposée dès le départ par Evelyne Saunier lors du travail de recherche mentionné note 6 . Il m'aura fallu quant à moi les quatre ou cinq années qui ont passé depuis, pour retrouver le principe de cette délocalisation, que je comprenais jusqu'alors comme le simple rapport de $\mathbf{x}$ à $\mathbf{y}$ : rapport qui ne relève pas nécessairement de la délocalisation loin s'en faut.
} 
interrompu ; l'autre qui explicite le concept de parcours et la forme de cette noninterruption, en mobilisant des éléments virtuels, que la notion évoque mais qu'elle ne met pas en place directement, et qui pourtant sont ce qui lui donne sa substance. On en propose la formulation suivante :

La notion linguistique de « fil » dit que :

$x$ est la manifestation continuée en un point $\boldsymbol{P}$, d'un parcours $X$ qui constitue le mode d'etre $Y$ $d^{\prime} y .^{20}$

Que $\mathbf{X}$ soit un parcours suppose que :

$X$ détermine et régule un mouvement virtuel $M$, pour un objet en mouvement virtuel $\boldsymbol{O}$.

Que la manifestation de ce parcours soit continuée en $\mathbf{P}$ suppose que :

$P$ est le point à partir duquel $M$ se déploie : le point où $M$ pourrait s'arrêter et où il ne s'arrête pas.

La nature précise de $\mathbf{o}, \mathbf{M}$ et $\mathbf{P}$, comme aussi leurs positions relatives par rapport à $\mathbf{x}$ et à $\mathbf{X}$ étant laissées indéterminées, cela constitue un des aspects de la variation que l'on peut observer d'un emploi à l'autre : la notion linguistique de « fil » dessine une forme qui peut se matérialiser de différentes façons suivant le contexte impliqué. Cette variation-là ne relève pas de principes généraux de variation, mais d'une simple affaire d'adaptation au contexte : il faut dans le contexte trouver ce qui va pouvoir tenir lieu de parcours, de mouvement, d'objet en mouvement et de point de non-retenue. C'est dire qu'elle dépend du contexte, et qu'elle ne permet pas d'expliquer en particulier pourquoi le mot admet plusieurs types de contextes bien différenciés. En revanche, on va voir que l'ambiguité de ce qu'il faut entendre par «manifestation », par "constitution» et par «mode d'être », qui concerne donc la première strate et les relations qui s'y structurent, induit des variations qui relèvent bien quant à elles de principes généraux, qui affectent la forme même de la

\footnotetext{
${ }^{20}$ On voit que dans cette formulation on ne dit pas la façon dont $\mathbf{x}$ et $\mathbf{y}$ sont mis en place : introduit via la position sujet du verbe pour $\mathbf{y}$ (y est donc donné comme étant ce à quoi le sujet réfère), posé par filer lui-même, par ce que filer dit, pour $\mathbf{x}$ ( $\mathbf{x}$ est donc un élément, qui peut ou non être un élément tangible, mais dont l'énoncé prédique l'existence). C'est que le fait que $\mathbf{x}$ soit posé tandis que $\mathbf{y}$ est introduit indépendamment, via le sujet, tient à quelque chose qui paraît général, à savoir la construction intransitive du verbe, ou plutôt le type de construction intransitive dont il s'agit: en l'occurrence une construction mettant en jeu un objet interne, et dans laquelle le terme en position sujet doit correspondre à ce dont le mode d'être se manifeste à travers ce que dit le verbe. Bref, cela tient au fait que filer est ici un certain type de verbe intransitif que l'on peut répertorier au moins en première approximation comme un inaccusatif (puisqu'il est question du mode d'être du référent du sujet, et non de ce qu'il pourrait déterminer) à objet interne. Autant dire que la dissymétrie entre $\mathbf{x}$ et $\mathbf{y}$ ne fait pas partie de ce qui fait la singularité de ce verbe particulier. Elle n'a donc pas sa place dans ce qui serait la caractérisation de la forme schématique du mot. Elle doit être donnée à un niveau de détermination différent, celui où le verbe se trouve associé à une catégorie grammaticale particulière, celle de verbe, et plus spécifiquement de verbe intransitif à objet interne et à structure de type inaccusative : voir donc le paragraphe 4.
} 
Syntaxe, référence et identité du verbe filer

configuration obtenue, et qui rendent compte effectivement de la variété des contextes observés.

\section{Déploiement des variations relatives au statut référentiel des entités impliquées}

On a indiqué au cours de la reconstitution opérée en 2. que les trois relations sur lesquelles s'ordonne la forme schématique étaient chacune susceptible de se concrétiser de différentes façons. Ce sont ces relations qui permettent de rattacher les uns aux autres, mais aussi de différencier les uns des autres, les quatre paramètres $\mathbf{x}, \mathbf{X}$, $\mathbf{Y}$ et $\mathbf{y}$ à travers lesquels on a montré que la notion de fil se décline. Selon la forme qu'elles prennent, on s'attend donc à avoir des agencements différents des paramètres en question: c'est dire que les variations qui sont là impliquées sont la source de véritables déformations de la configuration de base, propres à affecter les conditions d'emploi des mots concernés. En particulier, ces variations sont l'occasion pour les paramètres de se déployer dans leur différence, ou au contraire de se projeter les uns sur les autres dans une apparente coïncidence : c'est là un effet de kaléidoscope qui tient au fait qu'elles touchent fondamentalement, ainsi qu'on va le montrer, au statut référentiel des paramètres en question, selon qu'ils font effectivement fonction d'entités référentielles distinguées, ou qu'ils désignent une simple qualité qu'un autre paramètre actualise.

\subsection{Manifestations de phénomènes / manifestations de qualités}

La première relation est celle qui relie $\mathbf{x}$ et $\mathbf{X}$ : $\mathbf{x}$ est la manifestation de $\mathbf{X}$. Il se trouve que le concept même de manifestation est susceptible de recouvrir au moins deux types de configuration.

Ou bien $\mathbf{X}$ est un phénomène susceptible de se réaliser, et la manifestation est ce passage à l'acte par lequel $\mathbf{X}$ devient actuel. Dans ce cas $\mathbf{x}$ est simplement la forme actualisée de $\mathbf{X}$. Il ne s'agit donc pas à proprement parler d'entités distinctes : $\mathbf{x}$ et $\mathbf{X}$ sont une seule et même entité, sous sa forme actuelle ou sous une forme encore virtuelle. C'est dire que la manifestation est alors une relation unaire et réflexive : $\mathbf{X}$ s'est manifesté (et $\mathbf{x}$ n'est que $\mathbf{X}$ se manifestant).

Ou bien $\mathbf{X}$ est une qualité, et la manifestation désigne quelque symptôme de cette qualité, à travers laquelle elle devient manifeste. On a dans ce second cas une différence forte entre $\mathbf{x}$ et $\mathbf{X}$, qui ne relèvent pas du même registre puisque l'un est de l'ordre du factuel tandis que l'autre est de l'ordre de la propriété. Et la manifestation est bien cette fois une relation binaire : $\mathbf{x}$ manifeste $\mathbf{X}$. Un des points importants est que $\mathbf{x}$ et $\mathbf{X}$ peuvent alors se distinguer aussi sur le plan du contenu conceptuel (et non pas seulement en termes de type référentiel) : ainsi des faits aux contenus les plus variés peuvent attester du bonheur de quelqu'un, sans que ces faits doivent être par eux-mêmes ce que l'on voudrait décrire comme étant du bonheur; ce ne sont que des manifestations de bonheur.

En même temps, l'identité de contenu n'est pas pour autant exclue : il arrive que ce soit bel et bien des bêtises qui manifestent la bêtise de quelqu'un. Cela n'empêche une différence de statut référentiel: les bêtises sont des faits, 
correspondant à quelque événement ou quelque processus se déroulant ; la bêtise n'est même pas un fait virtuel, mais bien une qualité qui est réputée appartenir à un certain nombre d'êtres, et qui est censée permettre de classer ces êtres en catégories. Mais la qualité en question se trouve mise en relation avec un certain type de comportements, et ce sont justement de tels comportements qui se sont manifestés. Dans ce cas, la manifestation est en quelque sorte une relation transitive : $\mathbf{x}$ manifeste $\mathbf{X}$ en tant que $\mathbf{x}$ est du $\mathbf{X}$; ou, pour le dire autrement, $\mathbf{x}$ manifeste ce qu'il est ; car c'est en tant qu'il se trouve être de fait une occurrence de $\mathbf{X}$ que $\mathbf{x}$ vaut comme une manifestation de $\mathbf{X}$ dont il atteste. On voit que cela suppose que $\mathbf{X}$ soit une qualité d'un certain type : de celles qui correspondent à la mise en œuvre de faits, et qui peuvent par conséquent s'entendre comme décrivant des catégories de faits.

Cela définit donc un troisième cas de figure, où c'est bien une qualité qui se manifeste à travers un fait, mais en tant que ce fait se trouve être de fait le support de la qualité en question.

Ces trois interprétations jouent crucialement sur la question de savoir jusqu'à quel point $\mathbf{x}$ et $\mathbf{X}$ constituent ou non deux entités distinctes. Elles jouent aussi sur le statut référentiel de $\mathbf{X}$, qui est un fait (mais un fait ayant les qualités d'un fil), ou qui est d'abord une qualité (mais la qualité de faits), ou enfin qui est un fait exemplifiant une qualité (donc ici du fil, en tant que ce fil exemplifie les qualités propres aux fils). De telles distinctions, aussi brouillées soient-elles par la façon dont chacune se renverse pour retrouver l'autre (ainsi que l'indiquent mes parenthèses), sont néanmoins fondamentales pour une théorie de la référence : il s'agit d'opposer ce qui relève d'une logique extensionnelle ou quantitative ${ }^{21}$, et ce qui relève d'une logique intensionnelle ou qualitative; et cette opposition conduit à envisager des cas où les deux logiques coïncident, lorsque les qualités se définissent à partir des quantités qui les exemplifient $\mathrm{t}^{22}$. Si ce sont bien ces oppositions extrêmement générales qui sont en jeu dans l'ambiguité que nous repérons à propos du concept de manifestation, cela signifie qu'il s'agit là d'un effet particulier d'une variation qui, elle, serait générale entre le quantitatif, le qualitatif et ce qui se définit à la jonction du quantitatif et du qualitatif.

Or, il se trouve que ces trois interprétations de ce que peut recouvrir le concept de manifestation correspondent à trois grandes classes d'emploi pour filer (pour fil

\footnotetext{
${ }^{21}$ Comme il est d'usage dans la théorie culiolienne de la référence, on entend ici par quantités tout ce qui concerne les entités que la notion mobilise, par opposition aux qualités que cette notion peut exprimer, et qu'elle peut en particulier attribuer aux entités en question. Les qualités s'appliquent ou ne s'appliquent pas à des entités ; les entités ont des qualités. Une qualité ne s'actualise pas sans un support. Voir de Vogüé (1999) pour une présentation un peu détaillée de la façon dont entités et qualités peuvent s'articuler au sein des notions. Le point à comprendre est que ce que l'on appelle quantité dans ce cas ne prendra pas nécessairement une dimension numérique; la relation entre quantités/entités et numération est sans doute fondamentale mais elle est plus virtuelle, les entités étant définies sans doute par le fait qu'elles sont numérisables (mesurables, comptables, quantifiables par exemple), mais non pas par le fait qu'elles seraient directement des numérations.

22 Ainsi a-t-on coïncidence avec une notion comme la notion de « chat», parce que définir la qualité «chat» revient à donner le format de ce que peut être une entité chat. Avec une notion comme la notion de «blond», on aura au contraire dissociation, parce que les propriétés des entités auxquelles pourraient être attribuée la qualité «blond » sont pour l'essentiel totalement extérieures à la propriété en question.
} 
aussi sans doute, mais on n'a ici ni le temps ni l'espace de s'intéresser dans le détail à la variation de fil) : les emplois où il est question de prédiquer l'existence d'un fait (par exemple le fait que Mesrine ait filé), et où c'est donc ce fait qui est donné comme ayant la forme d'un fil ; les emplois où il est question de qualifier le fait que désigne $\mathbf{x}$ en lui attribuant la propriété d'avoir la forme d'un fil (par exemple dans Le temps a filé, où il s'agit simplement de décrire la façon dont le temps a passé); les emplois où il est question de prédiquer l'existence d'un fait qui consiste dans l'apparition d'entités ayant de fait la propriété d'être des fils (par exemple lorsque c'est le gruyère ou le caramel qui filent). Le fil comme événement, le fil comme qualité, le fil comme objet: la différence est sans doute difficile à reconstituer, mais on voit que les résultats sont bien différents. On conçoit en outre que les conditions d'emploi ne soient pas les mêmes selon qu'il s'agit de trouver un $\mathbf{y}$ dont le mode d'être soit constitué par un événement, ou un y dont le mode d'être soit une qualité, ou un y dont le mode d'être puisse être constitué par l'apparition d'objets fils.

\subsection{Constituant, constitué, constitutif}

Pour la relation de constitution qui lie $\mathbf{X}$ à $\mathbf{Y}$, on a déjà eu l'occasion de déployer les trois interprétations qui pouvaient en être données: $\mathbf{X}$ est un simple constituant de $\mathbf{Y}$ par exemple dans La lampe filait, où la forme effilée n'est qu'un aspect de l'allure que prend la lampe ; au contraire, $\mathbf{X}$ est ce qu'est $\mathbf{Y}$ dans Le train filait, où il s'agit de décrire le mouvement du train en lui attribuant la propriété d'être linéaire et ininterrompu; et $\mathbf{X}$ est un élément constitutif de $\mathbf{Y}$, quand il s'agit bien d'un constituant, mais que ce constituant s'avère déterminer entièrement le mode d'être de $\mathbf{y}$, comme par exemple dans Le fugitiff fila où le fait que la fuite soit ininterrompue est clairement un élément déterminant pour le mode d'être de ce fugitif.

On retrouve là un nouvel effet de la concurrence qui oppose référence quantitative et référence qualitative : dire que $\mathbf{X}$ est un constituant de $\mathbf{Y}$ revient à donner un statut quantitatif à $\mathbf{X}^{23}$; donner $\mathbf{X}$ comme figurant ce qu'est $\mathbf{Y}$ revient à donner un statut qualitatif à $\mathbf{X}$; donner enfin $\mathbf{X}$ comme un élément constitutif de $\mathbf{Y}$ revient à faire coïncider détermination quantitative et détermination qualitative, $\mathbf{X}$ ayant dans ce cas le statut d'une entité (un élément constitutif) qui a la particularité de fonder une qualité (de constituer $\mathbf{Y}$ : donc de le déterminer dans son être, donc dans ses qualités spécifiques).

C'est ainsi à nouveau un principe général qui rend compte de ce qui prend la forme de conditions d'emploi et de contraintes de sélection différentes. Par exemple, il faut que $\mathbf{y}$ soit une entité susceptible d'avoir un mode d'être déterminé par sa linéarité pour que $\mathbf{X}$ puisse faire figure d'élément constitutif de $\mathbf{Y}$ : c'est le cas du caramel, que les fils constituent ; et c'est le cas de l'oiseau dont le mode d'être quand il file est sans doute constitué par cette linéarité ininterrompue qui le fait disparaitre de

\footnotetext{
${ }^{23}$ La question est de savoir s'il est possible que $\mathbf{X}$ fasse fonction de qualité par rapport à $\mathbf{x}$ et de quantité par rapport à $\mathbf{Y}$. Autrement dit les deux sources de détermination du statut référentiel de $\mathbf{X}$ doivent-ils nécessairement coïncider? Il semble qu'il y ait des contraintes, mais que quelques discordances soient possibles, quand le statut d'un des termes se voit renégocié d'une relation à l'autre. On laisse ici cette question en l'état.
} 
ma vue. En revanche, le gruyère n'est pas constitué de fils quand il est fondu : il est simplement dans un état que l'on peut caractériser par la présence de fils ; la présence de fils fait là fonction de qualité, et non pas d'élément constitutif. De même le train, qui à la différence de l'oiseau, va seulement vite, mais ne disparait pas, a son mode d'être caractérisé par la forme fil, mais ne voit pas son mode d'être déterminé par cette linéarité ininterrompue.

On ne pourra pas dans le cadre de cet article envisager tous les cas de figure possibles. On voit cependant déjà comment cette opposition entre des traits caractérisants et des traits déterminants peut permettre de distinguer entre les cas où filer dit simplement la rapidité et ceux où il dit plutôt la disparition. Le troisième cas de figure, celui du trait constituant, plus descriptif, dirait alors simplement un élément de l'allure générale de $\mathbf{y}$ : ainsi le bas qui file, ou le pouls qui file sont sans doute mal en point, mais non pas pour autant disparus, ni même sujets à un mouvement rapide.

\subsection{Les aléas, l'être, l'existence : le dense, le compact, le discret}

La question des modes d'être a été examinée longuement en 2.5. On a pu en outre montrer que cette variation se manifeste à son tour à travers des types de contextes ou de co-textes différents : le fugitif advient dans son être de fugitif en filant, tandis que la rapidité de l'oiseau filant n'est que l'une de ses façons d'être possibles ; quant au chemin, s'il file, ce doit être une propriété et non pas une façon d'être, et ce n'est certainement pas ce qui lui confère son statut de chemin (il peut y avoir des chemins qui ne filent pas).

Il est facile de montrer comment cette dernière variation redéploie à nouveau la concurrence entre quantité et qualité : si $\mathbf{Y}$ est ce qui advient à $\mathbf{y}, \mathbf{Y}$ figure une façon particulière dont $\mathbf{y}$ s'actualise, et on est donc bien dans le registre des quantités; si $\mathbf{Y}$ est l'être d'y, $\mathbf{Y}$ est une qualité ; si $\mathbf{Y}$ est ce par quoi l'être d'y advient, on a affaire à une quantité actualisant une qualité d'y, ce qui signifie que qualité et quantité se trouvent articulées.

Ce ballet du qualitatif et du quantitatif prend cependant dans ce cas une dimension supplémentaire, que l'on ne trouvait pas dans les autres cas, qui est la dimension processive : quelque chose advient ou n'advient pas, où se joue ou non l'existence d'y. La variation en cause va prendre par conséquent une dimension aspectuelle (du moins pour ce qui concerne le verbe filer, cette variation se manifestant vraisemblablement pour le nom à travers l'opposition entre produit, support et objet du mouvement que l'on a vu en 2.6.). On retrouve en effet dans ce cas des oppositions à la Vendler entre des propriétés et des processus d'une part (l'être et l'aléa), entre des processus et des accomplissements d'autre part ('’aléa et le passage à l'existence). C'est dire que ces oppositions sont aussi celles qui sont décrites dans la TOPE à l'aide de la trichotomie du «compact», du «dense » et du «discret». Le «compact» est strictement qualitatif et réfractaire à toute problématique de l'actualisation : il correspond donc au cas des états de Vendler, et se caractérisera pas le fait qu'il correspond à des énoncés dans lesquels la glose explicitative en être en train de est exclue. Le «dense » est strictement quantitatif au sens où il correspond au contraire à une actualisation, dont nul être ne vient donner le format prédéfini : il s'agit donc de ce que Vendler appelle une activité, et elle se caractérise par son absence 
de finalité interne, ce qui correspond au fait que l'énoncé acceptera difficilement un circonstanciel de temps évaluant le temps pour finaliser le procès $\left(*_{e n}\right.$ trois minutes est donc exclu). Le "discret» articule quantité et qualité, et correspond aux accomplissements à la Vendler, la mise en coïncidence entre quantité et qualité figurant la finalité du processus mis en œuvre: il sera alors possible d'utiliser un circonstanciel de temps pour évaluer non pas la durée du procès mais le retard nécessaire pour finaliser ce procès (en trois minutes devient possible).

On dispose ainsi de critères pour classer les types de co-textes en fonction de ce dernier paramètre : on vérifiera que le caramel, le gruyère, le fugitif, le bas, le câble (Le câble a filé en dix minutes devrait être admis si l'on admet que filer décrit ici le temps que met le câble avant de se retrouver entièrement déroulé), et l'argent sont tous compatibles avec en (au passé composé); on vérifiera que le temps et le chemin sont peu compatibles avec la forme être en train de (comparez * Le temps est en train de filer et Ma vie est en train de filer). A partir de là on peut espérer reconstituer le détail de la variation à laquelle est soumis le filer intransitif ${ }^{24}$, en rapportant chacun des emplois recensés à l'une ou l'autre des configurations obtenues en combinant le jeu sur chacune des trois relations impliquées.

\subsection{Des possibles, des attestés et des figements}

Une telle reconstitution permettrait en outre de repérer quelles sont les combinaisons non recensées parmi les 27 (3×3×3) théoriquement prévisibles, et de déterminer les contraintes ou les contradictions qui peuvent départager le possible et l'impossible.

La difficulté cependant est qu'on n'a apparemment que peu de marges dans le choix des sujets possibles : si l'on compare avec d'autres verbes, la liste est tellement restreinte qu'il y a un sens à parler de locutions figées pour chacun des cas recensés. Cela signifie que pour rendre compte de l'existant, il faudra sans doute d'autres mécanismes explicatifs que celui des contraintes sur les configurations référentielles vues dans cette section. En particulier, le fait que les lexèmes en position sujet doivent pouvoir être interprétés comme mettant en œuvre un mouvement, avec un objet parcourant, et un point fixe de retenue est sans doute un élément explicatif important pour expliquer les lacunes observées : pour que le filer intransitif puisse cohabiter avec un sujet, il faut que d'une façon ou d'une autre ce sujet contienne en lui-même (dans sa propre figure) toute la figure de filer. Une forme de résonance entre les deux mots sur laquelle on va revenir ${ }^{25}$.

\footnotetext{
${ }^{24} \mathrm{Il}$ est possible qu'il y ait aussi un critère pour distinguer les trois interprétations de la relation de constitution: quand le $\mathbf{X}$ qui se manifeste est constitutif du mode d'être $\mathbf{Y}$, le verbe admet plus facilement le passé simple (Le fugitif fila / * son pouls fila / *son bas fila / * le chemin fila ou * le train fila, sauf s'il s'agit de dire que ce train disparaît); quand le $\mathbf{X}$ qui se manifeste est une qualité du mode d'être Y, le verbe admet plus facilement l'imparfait (Le train filait / Le chemin filait / l'argent filait / le câble filait / la lampe filait / * son pouls filait / * son bas filait). La raison de ces contrastes reste à préciser.

${ }^{25}$ C'est là encore Evelyne Saunier qui la première a mis ce fait de la résonance en évidence. Voir aussi Jalenques (2000).
} 


\section{Déploiement des variations syntaxiques}

\subsection{Nom et verbe : parties de discours}

\subsubsection{Schémas de lexis}

On a proposé d'associer à fil et à filer la même forme schématique : les deux mots se distingueraient à un autre niveau, à savoir celui des catégories grammaticales, où chaque catégorie se voit associée à un schéma grammatical particulier. C'est à ce niveau-là que $\mathbf{x}$ et $\mathbf{y}$ se trouvent éventuellement mis en correspondance avec des positions syntaxiques. Et c'est à ce niveau que se met en place l'opposition entre verbe et nom. La question est alors de comprendre ce qui peut faire la particularité d'un verbe par rapport à un nom.

L'hypothèse que l'on propose de faire, à la suite de Culioli, est que la particularité des verbes se joue dans le fait que ceux-ci s'entendent comme figurant le schéma organisateur d'un dire à constituer: on admet ainsi une conception très classique du verbe, selon laquelle ce serait dans le verbe que se jouerait la proposition. Culioli a proposé d'appeler lexis ce dire, qu'il conçoit non pas tant comme un contenu ou un «dictum» que comme un «à dire»: soit, une finalité, une visée, propre à engendrer une pluralité de dires effectifs que sont les énoncés s'employant, selon différents "modus ", à dire cet «à dire ». Il formule dès lors le lien entre verbes et lexis en posant que les verbes s'ordonnent nécessairement sur ce qu'il appelle un schéma de lexis, qui serait donc un outil pour structurer, à partir d'une forme schématique, une lexis.

L'objet du schéma de lexis est de conférer à la notion un statut prédicatif. Il peut atteindre cet objectif par différentes voies, dans la mesure où il y a des prédicats de différents ordres. Mais le principe est toujours d'introduire une structure prédicative, en distinguant deux positions, où se différencient un prédicat et son support, et en posant une opération de mise en relation de ces positions l'une par rapport à l'autre. On propose ici de noter le schéma de lexis : $<\mathbf{S}$ r $\mathbf{P}>$, dans lequel $\mathbf{S}$ désigne le support, $\mathbf{P}$ le prédicat, et $\mathbf{r}$ leur mise en relation.

Telle serait donc la différence entre filer et fil, le premier étant prédicatif et dissociant de ce fait un prédicat de son support, là où le second rassemble et ne distingue que diverses occurrences d'une notion englobante, qui tout à la fois recouvre ces occurrences et dit leur identité. Dissociation versus coïncidence, tel serait le fondement de l'opposition entre verbes et noms : cette opposition correspondrait par conséquent à l'intuition à la fois triviale et confuse selon laquelle le verbe dit tandis que le nom nomme ${ }^{26}$, ce qui implique que le verbe dise autre chose, tandis que le nom nomme ce qu'il se trouve effectivement désigner. Le reste, à savoir par exemple les effets de processualité, d'événementialité, d'instabilité, de transitivité et de transitionalité, qui peuvent arrimer le verbe au temps ou au faire, les questions de substance, d'essence ou d'individualité, qui peuvent arrimer le nom à l'être, serait alors à mettre au compte de variations d'une autre ordre. On aurait simplement deux schémas, le schéma de lexis, et parallèle à lui, ce qui serait un «schéma de

\footnotetext{
${ }^{26}$ Opposition élaborée collectivement par l'ensemble des participants à ce volume, lors des séances de travail du groupe.
} 
nomination », mobilisant non plus un support et un prédicat, mais quelque occurrence et une notion, avec une relation de repérage entre les deux (relation "epsilon») opérant l'identification de $\mathbf{o}$ en relation avec $\mathbf{N}:<\mathbf{o}$ « epsilon » $\mathbf{N}>$.

On voit que dans cette différence entre verbe et nom, c'est essentiellement le statut d'une position de sujet qui est en jeu, celle-ci se trouvant correspondre très exactement à ce qu'on a appelé le support : une entité autonome, dissociée a priori de la notion verbale, dont cette notion va néanmoins être prédiquée. L’idée serait donc que pour les noms il n'y a pas de sujet: pas de sujet à dire, donc aussi rien à faire advenir ; simplement, de manière en définitive toute circulaire, faire être ce que l'on dit être.

On traite donc ici les catégories grammaticales comme de véritables «parties du discours », avec l'idée qu'il faut de fait les envisager comme parties prenantes de l'activité discursive (et non pas comme résultats d'une catégorisation préalable du monde où il y aurait d'un côté des procès pour les verbes, de l'autre des entités pour les noms et aussi des qualités pour les adjectifs et encore des relations pour les prépositions, tous bien distinguables les uns des autres) ${ }^{27}$.

\subsubsection{Les types de procès}

A partir du schéma de lexis, comment rend-on compte du fait que le verbe puisse référer à un processus, ou à un événement, ou à un état transitoire, ou à un concept? On voit que ces différences essentiellement aspectuelles doivent pouvoir être mises en relation avec l'opposition entre les trois modes de référence distingués en 3. : référence quantitative, qualitative, ou articulant quantité et qualité.

Si le prédicat est associé à une référence quantitative, il s'agit de prédiquer l'existence d'un état de fait. On est alors dans une logique de la description, et la relation au sujet est extrêmement lâche, celui-ci étant seulement le support de cet état de fait, qui peut dès lors ne le caractériser que très partiellement.

Si le prédicat est associé à une référence qualitative, il s'agit d'attribuer une propriété au référent du sujet. On est bien dans une logique du jugement, et le sujet est l'objet de ce jugement.

Si le prédicat est associé à une référence à la fois qualitative et quantitative, il s'agit de prédiquer l'existence d'un événement, et on est dans une logique de la narration, où la présence de cet événement s'oppose en bloc à son absence.

Cette opposition correspond à trois grandes valeurs du présent. Dans le premier cas, le présent s'interprète comme un présent déictique, et l'énoncé décrit ce qui est en train de se produire dans la situation d'énonciation. Dans le second, le présent s'interprète comme un présent générique, l'énoncé décrivant une propriété du sujet qui se trouve déterminée indépendamment des paramètres temporels. Dans le

${ }^{27}$ Dans le modèle qui s'élabore ici, il faudrait que toute partie du discours soit caractérisée par un schéma spécifique : les thèses de Franckel et Paillard sur les prépositions qui toutes relèvent d'un repérage X R Y (voir par exemple Paillard, 2002) peuvent s'entendre comme des propositions de ce type, où il ne s'agit pas de décrire la forme générale des formes schématiques des prépositions, mais de fournir le schéma qui, indépendamment de la singularité de chaque forme schématique, dit la grammaire d'une préposition : c'est-à-dire la « partie » que joue dans le discours toute préposition. 
dernier, le présent s'interprète comme un présent narratif, référant à un passé ou à une fiction, avec des événements qui s'enchaînent les uns aux autres.

On constate que dans le cas du filer intransitif, le présent vaudra comme un présent déictique : qu'il s'agisse du caramel, de l'oiseau, du bas ou de ma vie c'est toujours au moment où je le dis, dans une parfois difficile coïncidence, que les fils se manifestent.

Il faut donc préciser dans la caractérisation de son schéma grammatical, qu'il relève d'un schéma de lexis à référence quantitative : il s'agit de décrire ce qui a lieu dans la situation d'énonciation, qui est un élément caractérisant en partie le sujet.

A partir de là, toutes les variations vues en 3. pourront se déployer, ordonnant des filer plus ou moins perfectifs et plus ou moins imperfectifs d'une part, avec des fils objets, qualités ou phénomènes, et avec une relation plus ou moins globale ou partielle entre le fil et le référent du sujet selon la façon dont s'interprète la constitution. Le seul point commun entre toutes ces variantes étant le caractère descriptif et déictique de l'énoncé comportant filer.

\subsection{Les structures de complémentation}

On a proposé dans la section 1. de distinguer un troisième niveau dans ce qui fait l'identité lexicale, à côté de la forme schématique et du schéma grammatical, ce troisième niveau spécifiant ce qui caractérise à proprement parler la distribution du mot, et donnant dès lors l'ensemble des structures de complémentation dans lesquelles le mot peut s'intégrer. Pour filer, on aura donc l'ensemble de ce que l'on a appelé son répertoire, c'est-à-dire, outre la structure intransitive, une structure transitive, une structure locative et une structure attributive.

Le problème, pour rendre compte de cette dimension-là de la variation, est d'expliquer comment on obtient ces différentes structures à partir du schéma de lexis de base $^{28}$. Cela suppose que l'on parvienne à reconstituer des principes de construction de ces structures.

A priori les structures en question se présentent comme des chaînes de compléments, où sont mentionnés tous les éléments qui apparaissent pour compléter le mot, dans l'ordre où ils apparaissent si ordre il y a, et avec les prépositions qui les introduisent le cas échéant: Culioli note $\mathrm{Co}, \mathrm{C}_{1}, \ldots$. Cn ces chaînes, les indices ne servant en l'occurrence qu'à ordonner, et distinguer les uns des autres, ces compléments. Il est à noter que de ce point de vue, le sujet est un complément comme les autres pour son verbe, puisqu'il fait partie des éléments nécessaires pour le compléter. C'est seulement le premier des compléments, et on signale seulement son caractère périphérique en le notant $\mathrm{Co}$ au lieu de $\mathrm{C}_{1}$. Sa particularité de sujet, comme ce qui l'oppose aux autres compléments, tient de sa position au niveau du schéma de lexis, et non au niveau de la chaîne de complémentation. De manière générale, ces

\footnotetext{
28 On explique comment on obtient les structures en question, on ne cherche pas à expliquer pourquoi ce sont ces structures et non pas d'autres qu'on obtient : il n'y a aucune raison à ce qu'il y ait une motivation dans ce domaine dans la mesure où a priori une unité peut être indéfiniment reconstruite, que ce soit en discours, ou en diachronie. Voir en particulier la construction signalée note 1.
} 
chaînes sont strictement linéaires, et ne présument aucune structuration particulière : il faut en effet qu'elles soient compatibles avec plusieurs modes de combinaison.

De fait, les structures dans lesquelles les mots s'intègrent sont elles-mêmes le lieu de modalités de construction et d'interprétation variées : il y a plusieurs catégories de structures intransitives (absolutives, ergatives, inchoatives par exemple), il y a plusieurs catégories de structures transitives (voir ci-dessous), et les prépositions sont sujettes à des variations aussi importantes que celles des lexèmes étudiés. Il faut donc ajouter aux chaines de complémentation une description des modalités d'inscription des lexèmes dans ces chaînes.

Ces modalités sont diverses, mais ne sont pas pour autant indéfiniment variables: il y a plusieurs catégories de structures transitives, il n'y en a pas une infinité ; et on ne dira pas qu'il y a autant de formes de transitivité qu'il y a de verbes transitifs, pas plus qu'on ne dit qu'il y a autant de valeurs de filer qu'il y a d'énoncés comportant ce verbe. On recherche donc des règles précises rendant compte des constructions attestées et de leur mode de construction.

Par rapport à des dérivations transformationnelles classiques, ces règles procèdent par ajout (ajout d'élément extérieur) et par combinaison (combinaison avec un système de positions extérieur): cela signifie qu'elles procèdent par complexification au lieu de travailler à contenu lexical et interprétatif constant. Elles ont en outre la particularité de mettre en jeu non pas seulement des principes de construction généraux, mais des éléments, singuliers : telle préposition, tel type de complément, telle position.

La théorie des formes schématiques travaille depuis un certain nombre d'années sur les modalités de ces combinaisons, qui consistent à articuler l'une à l'autre deux unités a priori autonomes. Il a été proposé de distinguer trois types de combinaison, qui sont respectivement l'ajout (appelé encore, peut-être plus proprement, enchâssement), le nouage (ou chevauchement) et la greffe (ou superposition $)^{29}$. On part de l'idée que toutes les unités sont fondamentalement relationnelles (à cause des schémas grammaticaux qui les inscrivent nécessairement dans des schémas relationnels). L'ajout correspond au plus faible niveau d'intrication entre les deux relations combinées : l'une des relations prend l'autre comme argument, et la met donc en relation avec autre chose. Le nouage correspond au cas où l'un des termes de la première relation est aussi impliqué dans la deuxième relation, articulant ce faisant les deux relations l'une à l'autre. La greffe correspond au cas où les deux relations se superposent, chacune venant dès lors apporter à l'autre sa propre configuration (voir par exemple Jalenques (2002) et Paillard (2002) pour des mises en application de ce programme à des combinaisons entre verbes et préfixes).

On propose d'utiliser ce modèle pour rendre compte de la façon dont une structure transitive se construit à partir d'une structure intransitive.

${ }^{29}$ Voir Paillard (2000) et Jalenques (2000). 


\subsubsection{De l'intransitif au transitif: causation, transitivation, spécification}

En droit, le fait de passer d'une structure intransitive à une structure transitive peut consister à rajouter un sujet ou bien à rajouter un objet. Par exemple, à partir de l'intransitif vivre, on construit des transitifs comme vivre sa vie, vivre cent ans, vivre un moment inoubliable, vivre son couple, en ajoutant un complément $C_{1}$ qui pourra être articulé de diverses façons avec la relation marquée par le verbe intransitif (il faudrait arriver à reconstituer ce qui relève de la greffe, ce qui relève de l'ajout, et ce qui relève du nouage dans les exemples cités). En revanche, pour filer, le passage à la structure transitive consiste à rajouter un complément Co. Ce complément Co s'inscrit luimême dans une relation, en tant que sujet du schéma de lexis. La question est de savoir comme cette relation va se combiner avec celle que construit filer en tant qu'intransitif.

Si la combinaison procède d'un ajout, cela signifie que Co est donné comme le support d'une prédication dont le contenu est donné par le filer intransitif: le résultat d'une telle combinaison correspondra par conséquent soit à une causation $(<$ Co fait que $<\mathrm{C}_{1}$ file $\left.>>\right)$, soit à une forme de localisation ( $<$ Co a $<\mathrm{C}_{1}$ file $\left.>>\right)$, soit enfin à une attribution (<pour Co il est vrai que $<\mathrm{C}_{1}$ file $>>$ ). Et l'on voit que cela dépendra du caractère discret, dense, ou compact du schéma de lexis dans lequel est pris Co.

Si la combinaison procède par nouage, cela signifie que $C_{1}$ se trouve à la fois pris dans une relation à $C_{0}$ et dans sa relation à filer: le résultat correspondra par conséquent soit à une transitivation effective $\left(<\mathrm{Co}\right.$ fait $\left\langle\mathrm{C}_{1}\right\rangle$ file $>$ ) où c'est le résultat de l'action de Co sur $\mathrm{C}_{1}$ qui produit le filage, soit à une localisation transitive $(<\mathrm{Co} a$ $<\mathrm{C}_{1}>$ file $>$ ), soit à une attribution transitive ( $<$ pour Co, il est vrai $<$ que $\mathrm{C}_{1}>$ file $>$ ).

$\mathrm{Si}$ enfin la combinaison procède par greffe, les deux relations superposées doivent avoir le même Co, et on obtient $\quad<<\mathrm{Co}$ file $>\mathrm{r} \mathrm{C}_{1}>$, ce qui correspond à une détermination via $C_{1}$ de ce qui serait l'objet interne du filage.

On obtient par conséquent en droit neuf possibilités. Il semble que cela corresponde à peu près à ce que l'on observe, quoique le détail soit plus obscur qu'on ne le voudrait au moins dans les cas où Co n'est ni un agent (c'est-à-dire inscrit dans un schéma de lexis événementiel), ni un localisateur (c'est-à-dire inscrit dans un schéma de lexis descriptif), mais un thème (c'est-à-dire inscrit dans un schéma de lexis de type thème/propos). Pour le reste, on trouve les cas suivants :

Ajout :

Causation : filer la laine, du verre, la pièce, un câble, un son, une métaphore, nos destins = Co fait que la laine, la pièce, le câble, la vie, les destins, etc. filent (pour une laine, une pièce, etc... qui ne filent pas sans qu'on les file)

Localisation : filer son bas

$=$ Co a son bas qui file 
Syntaxe, référence et identité du verbe filer

Nouage :

Transitivation : filer sa toile, sa corde, ses habits, sa dot, nos destins

= Co fait que la toile, la vie, les destins soient filés

Localisation transitive : filer le parfait amour

= Co a le parfait amour qui est filé

Greffe :

Détermination télique : filer quelqu'un

$=$ Co fait à $\mathrm{C}_{1}$ que Co file

Détermination quantitative : filer trois nouds

$=$ Co a trois nœuds que Co file

L'absence de valeur de type thème/propos est peut-être une particularité de filer qui, de la même façon qu'il se cantonne aux références quantitatives dans sa forme intransitive, se limiterait aux références discrètes ou denses pour sa forme transitive.

Le mécanisme permet par ailleurs d'expliquer le fait que l'expression filer sa vie soit ambiguë, selon qu'on l'interprète comme signifiant « transformer sa vie en fil », ce qui revient à la défaire, ou "fabriquer sa vie à l'aide de fils", ce qui revient à la construire. Chateaubriand joue sans doute de cette double interprétation lorsqu'il écrit : «Le temps file notre fragile vie comme on file le verre ».

\subsubsection{Les locatifs et les attributifs: diversification des constructions}

Que filer s'interprète comme un verbe de mouvement avec un groupe prépositionnel de lieu peut être tenu comme la résultante de trois faits : 1) la notion linguistique de « fil » implique de manière générale la mise en œuvre d'un mouvement ; 2) le verbe intransitif filer possède lui-même toute une série d'emplois dans lesquels il s'interprète comme un verbe de mouvement (Mesrine a encore filé); 3) le groupe prépositionnel s'interprétant comme un circonstanciel de lieu, il oriente filer dans la direction du déplacement. Autrement dit, on admet que le groupe prépositionnel sélectionne parmi les diverses valeurs possibles de filer celle qui paraît la plus compatible avec l'interprétation première de ce groupe. Cela a pour conséquence que les deux effets de sens vont cohabiter : il est dit que le référent du sujet se déplace et il est dit qu'il y va sans retard, qu'il est rapide, et qu'il disparaît donc vite de la vue du locuteur. Dès lors il y a un sens à considérer ici que verbe et préposition se greffent l'un sur l'autre, la notion linguistique de «fil» venant en quelque sorte donner corps au déplacement marqué par la préposition.

Le même type d'analyse peut être appliqué au cas de la construction attributive : c'est cette construction qui en elle-même prend une valeur causative (voir la démonstration générale de cette propriété dans Yaguello 1998), et il faut juste expliquer que cette valeur soit un «faire avoir » plutôt qu'un «faire voir» (comme dans montrer quelque chose à quelqu'un) ou un "faire savoir» (comme dans apprendre quelque chose à quelqu'un). Que ce soit la structure $\mathrm{V} \mathrm{C}_{1}$ à $\mathrm{C}_{2}$ qui configure la valeur générale de causation, signifie que cet emploi relève à nouveau d'une greffe : étant 
donné le cadre causatif fourni par ce schéma, filer vient redessiner cette causation à l'aide de sa figure, conférant dès lors à la causation une valeur de transmission.

Il est à noter que ces deux emplois ont pour particularité d'être des emplois argotiques. C'est le cas aussi du filer transitif qui signifie «suivre quelqu'un». On peut penser que l'argot, de par son caractère figé d'une part, de par son caractère métaphorique d'autre part, est le lieu par excellence de la figure de la greffe.

Il faudrait étudier dans le détail quels sont les types de $C_{1}$ qui peuvent intervenir dans cette construction attributive : on a des microbes, des coups et des émotions, et on a des objets si tant est que ces objets étaient déjà attendus, ou si tant est que le don est provisoire (Tu me files ta bagnole ?). De fait filer consiste à mettre $\mathrm{C}_{1}$ en mouvement à partir d'un point de non-retenue («je te file ce que je ne retiens pas pour moi »); il ne s'agit donc pas de changer simplement de localisateur pour $\mathrm{C}_{1}$; il s'agit de ne pas retenir $\mathrm{C} 1$ et de le laisser aller sur un parcours prédéfini.

On voit que c'est bien une théorie de la transmission et une théorie de la circulation que filer déploie ici, avec ses moyens linguistiques : quand les mots servent à théoriser le monde.

\subsubsection{Le figement lexical : quand filer se greffe sur un mot}

On a mentionné en 3.4. le caractère figé des emplois du filer intransitif. Cela vaut aussi bien pour les autres constructions, où l'on retrouve des listes de possibles extrêmement contraintes.

Il est possible de donner une explication à ce figement: la relation entre le verbe et les items lexicaux qui lui servent de complément procéderait elle aussi de la greffe, le mot sujet ou objet venant se greffer sur filer pour lui donner sa substance, et réciproquement filer venant réinterpréter avec sa forme schématique le mot en position sujet ou objet pour y inscrire parcours, point fixe, manifestation, constitution et mode d'être.

Si cette explication est correcte, cela signifie qu'il est possible d'envisager en droit trois types de relation entre les verbes et leurs compléments : le complément peut être un ajout, ou peut être noué au verbe, ou peut être greffé sur le verbe, ce qui conduit à ces effets de figements où verbe et complément entrent en résonance. Qu'il $\mathrm{y}$ ait des verbes plus propices à la greffe, d'autres plus propices au simple nouage, d'autres procédant par ajout, n'est pas exclu. Mais cela pose la question de ce qui peut séparer ces types de verbes. Qu'est-ce qui rend filer propice à la greffe ? Qu'est-ce qui fait que tel verbe ou tel mot est plus qu'un autre susceptible d'appeler ces figements ? Peut-être est-ce là ce qui caractérise les verbes qui comme filer sont "concrets», embarquant avec eux une portion du monde, pour l'installer dans la phrase. 
Syntaxe, référence et identité du verbe filer

\section{RÉFÉRENCES BILBIOGRAPHIQUES}

BENVENISTE, E., 1954, "Problèmes sémantiques de la reconstruction », in Problèmes de linguistique générale, 1 (1966). Paris : Gallimard, 289-307.

BENVENISTE, E., 1964, "Les niveaux de l'analyse linguistique », in Problèmes de linguistique générale , 1 (1966). Paris, Gallimard, 119-131.

CADIOT, P., LEBAS, F., 2003, «Monter et la constitution extrinsèque du référent », Langages, $150,9-30$.

CULIOLI, A., 1978, «Valeurs modales et opérations énonciatives », in (1990) Pour une linguistique de l'énonciation, 1. Paris, Ophrys, 135-155.

CULIOLI, A., 1987, «Formes schématiques et domaine», in (1990) Pour une linguistique de l'énonciation, 1. Paris : Ophrys, 115-126.

DE VOGUE, S., 1999, "Construction d'une valeur référentielle : entités, qualités, figures », Travaux linguistiques du Cerlico, 12. Presses Universitaires de Rennes, 77-106.

DE VOGUE, S., 2001, «L'épilangue au pied de la lettre », Le Gré des langues, 16. L’Harmattan, $9-48$.

DE VOGUE, S. et FRANCKEL, J.-J., 2002, «Identité et variation de l'adjectif grand », Langue française, 133, 28-42.

FRANCKEL, J.-J., 2002, «Introduction: Le lexique, entre identité et variation», Langue française, 133, 3-15.

JALENQUES, P., 2000, Contribution à l'étude du préfixe RE en français contemporain : pour une analyse compositionnelle du verbe regarder. Thèse de doctorat, Université Paris 7, Chapitre 8.

JALENQUES, P., 2002, «Etude sémantique du préfixe re- en français contemporain : à propos de plusieurs débats actuels en morphologie dérivationnelle », Langue française, 133, 74-90.

KLEIBER, G., 1999, Problèmes de sémantique, la polysémie en questions. Lille : Presses Universitaires du Septentrion.

PAILlARD, D., 2000, «A propos des verbes "polysémiques»: identité sémantique et principes de variation », Syntaxe et sémantique, 2, 99-120.

PAILLARD, D., 2002, "Contribution à l'étude du préfixe sous- combiné avec des bases verbales », Langue française, 133, 91-110.

SAUNIER, E., 1996, Identité lexicale et régulation de la variation sémantique : contribution à l'étude des emplois de mettre, prendre, passer et tenir. Thèse de doctorat, Université Paris X.

YAGUELLO, M., 1998, «La relation de causativité dans les constructions attributives du français », Le Gré des langues, 14, 42-61. 